\title{
Minutiae Fusion Based Framework for Thumbprint Identification of Identical Twins
}

\author{
Kamta Nath Mishra ${ }^{1}$ \\ Department of Computer Science \& Engg., B. I. T. Mesra, Ranchi (Allahabad Campus), India \\ E-mail: mishrakn@yahoo.com \\ P. C. Srivastava ${ }^{2}$ \\ Department of Computer Science \& Engg., B. I. T. Mesra, Ranchi (Allahabad Campus), India \\ E-mail: prakash_bit123@rediffmail.com
}

\author{
Anupam Agrawal ${ }^{3}$ \\ Department of Information Technology, Indian Institute of Information Technology, Allahabad, India \\ E-mail:anupam69@gmail.com \\ Rishu Garg ${ }^{4}$, Ankur Singh ${ }^{5}$ \\ Department of Computer Science \& Engg., B. I. T. Mesra, Ranchi (Allahabad Campus), India \\ E-mail: rishug18@gmail.com, arvindankur.singh@gmail.com
}

\begin{abstract}
Identical twins identification is a challenging task because they share the same DNA sequence. This research paper presents minutiae coordinates and orientation angles fusion based technique for thumbprint identification of identical twins. Six different thumbprint images of identical twins were taken at a fixed time interval using H3 T\&A terminal. The minutiae coordinates and orientation angles of these thumbprints were fused to form a union set. The union set values were stored in the smartcard memory for further identification.
\end{abstract}

The minutiae coordinates and orientation angles of a thumbprint of the person to be identified are computed and fused together for online identification. The fused minutiae are compared with the minutiae union set values stored in the smartcard memory for identity verification. The proposed method was tested on a self generated identical twin dataset and 50 identical twins of standard FVC04 and FVC06 datasets. We observed in experiments that the proposed method is accurately differentiating the identical twins of self generated and FVC datasets.

Index Terms - Minutiae Coordinates, Orientation Angles, Minutiae Union Set, Identical Twins, and Thumbprint Patterns

\section{Introduction}

Soft Computing is a tool which deals effectively with uncertainty, vagueness, and imprecation and it provides a low cost solution. It plays an indispensable role in computational engineering. Soft computing provides us an option for finding optimal solutions which are unpredictable, uncertain, and partially true. Many soft computing techniques (Artificial Neural Networks, Genetic Algorithms, Fuzzy Logic Models, and Particle Swarm Techniques etc.) are used in Biometric systems. We can determine the partial true value of binary set depending upon completeness or incompleteness of results by using fuzzy logic model whereas artificial neural networks are designed for pattern reorganization and data classification. Fuzzy set can be used for storing the minutiae coordinates and orientation angles in the form of rough set or trained set which will be useful for identifying identical twins. Chen has used fuzzy set for storing the local triangle features of a thumbprint $[4,32]$.

Neurons are the biological elements which are used for information processing in the brain. Neural network approach was first introduced by Leung in 1990 [20]. A neural network consists of a number of heads or tails where heads are the nodes and tails are the links. The neural network provides interconnection between nodes [21]. Biometric system is a method used for identifying a person on the basis of physical and behavioral characteristics. Physiological features based biometrics include iris image, finger prints, thumb print, palm print, face, finger veins, hand geometry, DNA sequence, and palates. Behavioral features based biometrics includes gaits (style of walking and style of talking), signature, body language, hand writing, and voice [31].

Twins are classified in two categories: fraternal twins and identical twins. We can easily identifying the 
fraternal twin because they differ in face structure, DNA sequence, and genetic compositions. Identical twins are produced from the same egg. Therefore, they share same DNA sequence and face structure [28, 29]. Identical twins can be identified on the basis of their thumbprints. A thumbprint image includes bifurcation points, ridges, left loop, right loop, arc and end points . These features of thumbprints play very important role in identical twins identification [11].

This paper classifies identical twins identification in two phases:

Phase 1: Train the thumbprint data sets using soft computing techniques.

Phase 2: Authenticate the person using a predefined process.

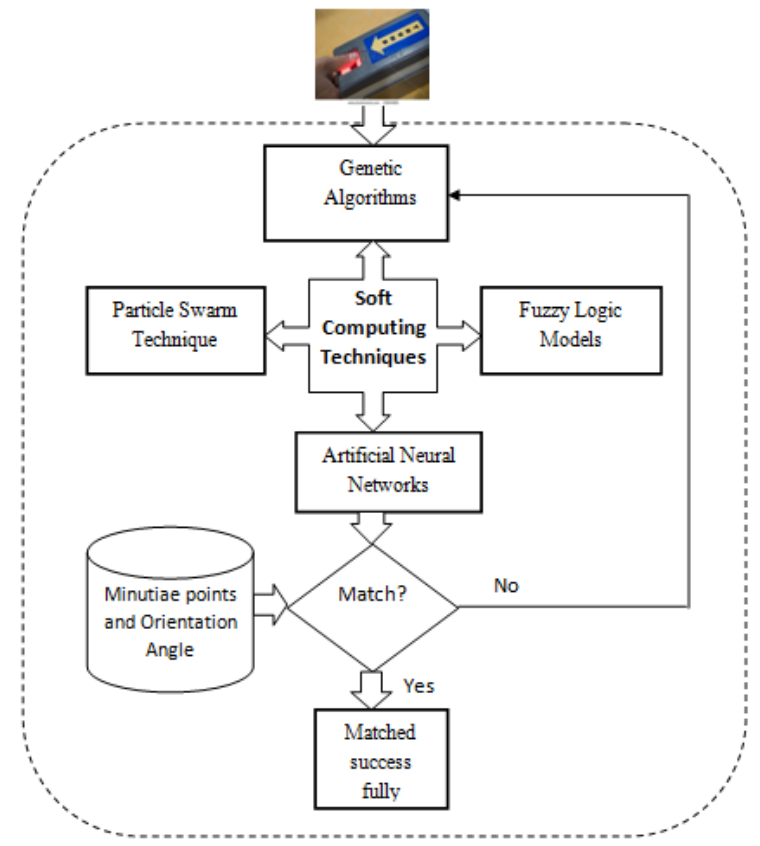

Fig. 1: Represents the soft computing techniques used by researchers for identifying an identical twin

The breakage points and bifurcation points of a thumbprint are called as minutiae points. The orientation angle of a minutia point is called as minutia orientation angle. This research paper uses minutiae coordinates and orientation angles fusion based fuzzy set technique for identification of identical twins. In the next section (section II) we have discussed the literature review related to identical twins identification. The section III describes a mathe matical model of minutiae fuzzy set based thumbprint identification method. In section IV of this paper we have proposed a novel minutiae fusion based union set technique for classifying identical twins. The section V discusses the experimental analysis of proposed method on self generated and standard datasets. The section VI describes conclusions and future research work.

\section{Literature Review}

Jung proposed a robust fingerprint identification method based on hybrid pattern recognition system for identifying identical twins. The structural characteristics of a thumbprint are fetched and then fuzzy set is applied to the extracted features for identification. Finally, the result is determined on the basis weighted matching scores of quantitative extracted features [16]. Chen proposed an algorith $m$ for distorted fingerprint matching on the basis of local triangle feature set. He represented a fingerprint by fuzzy feature set and local triangle feature set. The similarities of fingerprints were measured on the basis of fuzzy features and local triangle features [4, 5]. Mudholkar developed an effective authentication technique for fingerprint recognition using intrusion detection system. Mudholkar proposed a new algorithm which includes two basic steps (enrollment and authentication processes) for identifying identical twins [25].

Indi proposed a new technique for identifying a person using Personal Unique Identification System (PUIS). The PUIS includes pre-processing, binarization, thinning, minutiae detection, minutiae extraction, and minutiae feature matching steps [11]. Sun proposed a multi-biometric system for identifying identical twins and he proved in his research that a multi-biometric model is better in terms of robustness and accuracy in comparison to a single feature based biometric system [28].

Anil K. Jain compared the performance of single biometric and multi-biometric systems and concluded that a multi-biometric system can overcome the bottlenecks of a single biometric system [12]. He proposed a ridge and minutiae patterns based technique for thumbprint verification of identical twins [13, 14]. Kulkarni proposed a minutiae orientation fields based fingerprint verification system. Kulkarni used two databases; University of Bologna and Biometric Laboratory databases for testing the proposed algorithm. The proposed method showed better GAR (Genuine Acceptance Rate), low FAR (False Acceptance Rate), and low computational complexity [19].

Chen T. P. proposed that magnetic strip cards are no more required for authentication purpose because we have venerable microprocessor technology (smart card) which can be used for developing fingerprint based authentication system [3].

Krishneswari $K$. calculated the performance of multimodal biometrics using Discrete Cosine Transform (DCT) and information gain [17]. Tao X. proposed P071 and SDK verifinger algorithms for identical twins identification. Tao used 83 twin pairs for evaluating the performance and he observed that his algorithms are clearly distinguishing identical twins on the basis of their fingerprints [30]. Chen proposed a normalized fuzzy similarity measurement algorith $\mathrm{m}$ for distorted fingerprints recognition. The algorithm 
includes two steps namely fingerprint alignment based topological score computations, and similarity matching between the templates and input fingerprints $[4,5]$.

Velamuri recognized the fingerprints using a fuzzy inference technique. The method uses template creation and fingerprint matching steps [32]. Iancu introduced a fuzzy logic based technique for fingerprint identification. In this technique Iancu compared two matching sets and selected the optimal from these using a fuzzy reasoning system [10]. Bennet proposed a fuzzy set measurement method for fingerprint recognition. The proposed method is based on discrete wavelet transform (DWT) and singular value decomposition (SVD) approaches [2]. Homem proposed a new methodology for mobile phone user identification using fuzzy fingerprints. He used filtered space saving (FSS) algorithm which uses bitmap counter. The bitmap counter values are determined by hash function [7].

Kondekar proposed a new method for fingerprint recognition system which is based on Extended Fuzzy Hyper Line Segment Neural Network (FHLSNN). Kondekar used finger code feature extraction technique for extracting the fingerprint features [18]. Bazan proposed a roughest based behavioral pattern identification system. He used a method called online elimination of non relevant parts for online reduction of irrelevant objects to identify a behavioral pattern [1]. Meva proposed a neural network based new approach for fingerprint recognition technique [21].

Josphineleela proposed an efficient fingerprint based attendance system. In this research paper Josphineleela presented a method for overcoming with buddypunching a well known loophole of traditional attendance system. $\mathrm{He}$ used novel fingerprint reconstruction algorith $m$ to reconstruct the image from minutiae and then change it to a gray scale image. Evaluation of the proposed algorithm is based on success rates of type $-\mathrm{I}$ "matching sample with original" and type -II "matches reconstructed image with different impression of original finger print images" attacks [15].

Patil D. S. proposed an input image and a query image based minutiae matching technique for fingerprint recognition. The method was tested for FAR and FRR to evaluate the performance of a fingerprint recognition system[26].

\section{Mathematical Background of Minutiae Fusion Based Universe Of Discourse}

Let $\mathrm{Z}$ be a fixed nonempty set to be called as the universal set. We can define it as:

$f: Z \rightarrow\{0,1\}$

Here, $\mathrm{f}$ is a characteristic function.
Let's take n samples of thumbprints for each person of identical twin pairs. These samples can be mathematically represented as:

$$
\text { Person_1_Twin } 1=\left\{f_{a 1}(x), f_{a 2}(x), . . . . f_{a n}(x)\right\}
$$

Here, $f_{a 1}(x)$ represents first sample, $f_{a 2}(x)$ represents second sample, and $f_{a n}(x)$ represents $\mathrm{n}^{\text {th }}$ sample of first person of first identical twin pair.

A minutia point consists of three values: $X$ coordinate, Y coord inate, and orientation angle $(\theta)$. The directions of a minutia orientation angle can be categorized into eight parts namely north $(\mathrm{N})$, northeast (NE), east (E), south-east (SE), south (S), southwest (SW), west (W) and north-west (NW). If each direction of a minutia point is represented by a number then a minutia point and its orientation angle can be represented by following mathematical equations:

Minutia Point $=(X, Y, \theta)$;

Where, $\theta$ is the angle of orientation of a minutia point.

$$
\begin{aligned}
\theta & =\{N, N E, E, S E, S, S W, W, N W\} ; \\
\Rightarrow \theta & =\{1,2,3,4,5,6,7,8\} ;
\end{aligned}
$$

The identical twins can be identified on the basis of fusion of minutiae coordinates and orientation angles using following three steps:

Step 1: Create a set $F_{U}$ (where $F_{U}$ is the union of the thumbprints).

In this step, we have taken the union of minutiae coordinates with orientation angles of first $n$ thumbprints of a pers on of the identical twin pair. This union can be represented by following equation:

$$
F_{U}=A_{1} \cup A_{2} \cup \ldots \cup A_{m}
$$

In abbreviated form equation (2) can be written as:

$$
\bigcup_{i=1}^{i \rightarrow n} f_{a i}=f_{a 1} \cup f_{a 2} \cup \ldots \cup f_{a n}
$$

Therefore, we can now say that $\mathrm{F}_{\mathrm{U}}(\mathrm{x})$ is a union set which contains the union of minutiae coordinates and orientation angles of $n$ thumbprints of a person of the identical twin pair. Here, value of ' $n$ ' may vary from two to six.

Step 2: Take the input thumbprint image for identity verification of identical twin.

In this step, we will scan at least two thu mbprints for the first person and two thumbprints for second person 
of the identical twin. It can be mathematically written as:

$$
\begin{aligned}
F_{A} & =\left\{T A_{1}, T A_{2}\right\} \\
\text { and } F_{B} & =\left\{T B_{1}, T B_{2}\right\}
\end{aligned}
$$

Here, $\mathrm{TA}_{1}, \mathrm{TA}_{2}$ are the newly scanned thumbprints of first person and $\mathrm{TB}_{1}, \mathrm{~TB}_{2}$ are the newly scanned thumbprints of second pers on of the identical twin pair.

\section{Step 3: Thumbprints Verification.}

We know that $U$ is a union set which contains all the minutiae coordinates and orientation angles of first $n$ thumbprints of a person where $2 \leq n \leq 6$. Therefore,

$$
\bigcup_{i=1}^{i \rightarrow n} f_{a i} \geq f_{a i}
$$

The relationship between a union thumbprint set and a newly scanned thumbprint can be represented by figure 2 .

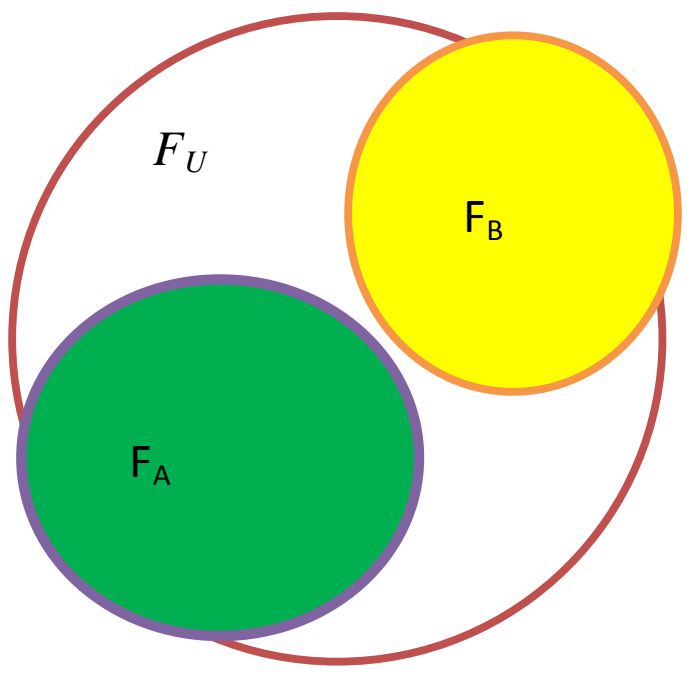

Fig. 2: Union set representation of minutiae coordinates and orientation angles of thumbprints

\section{Proposed Methodology}

Identical twins can be identified accurately with the help of their thumbprint patterns but the verification will be more authentic if we compare a single thumbprint sample with the union of thumbprints. The thumbprints for identical twin pair of figure 3 were taken using a scanning device (H3 T \& A terminal) from Sachcha Adhyatma Sanskrit Mahavidyalay, Sachcha Baba Ashram, Arail, Allahabad, Uttar Pradesh, INDIA, Phone No.: 0091-9452367236.

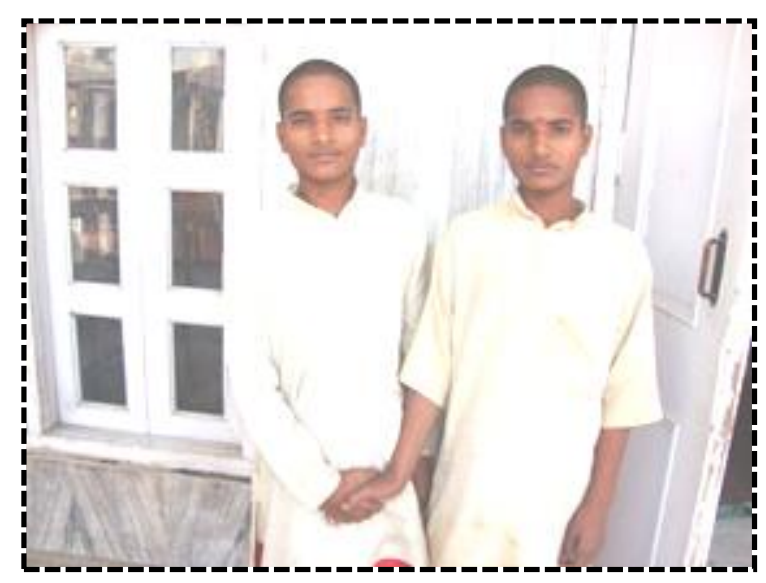

Fig. 3: Facial images of identical twin pair Servesh and Dhermesh

The proposed methodology uses three phases (A to $C$ ) for verifying the identity of identical twins.

\subsection{Preprocessing Phase}

A scanned thumbprint image is given as input to the preprocessing phase. An input image (raw image) is converted to a thinned binary image in preprocessing and it also includes following steps for converting thinned input:

Step 1: Image Smoothing [Join the broken ridges of inner parts of a thumbprint image.]

Step 2: Ridge Thinning [Decrease the thickness of ridges to a single pixel value using bwmorph( ) function of MATLAB 7.0.]

Step 3: Black and White Binary Image Conversion [Convert the thinned image to a binary image using im2bw ( ) function of MATLAB 7.0]

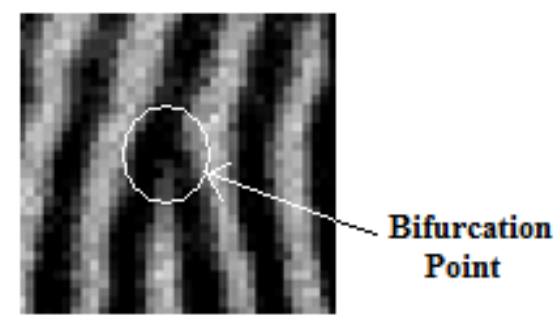

Fig. 4(a)

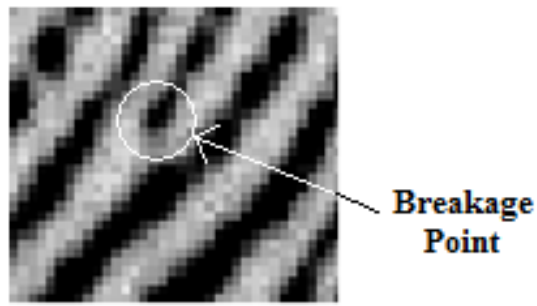

Fig. 4(b)

Fig. 4: The ridges of a thumbprint image [22] 

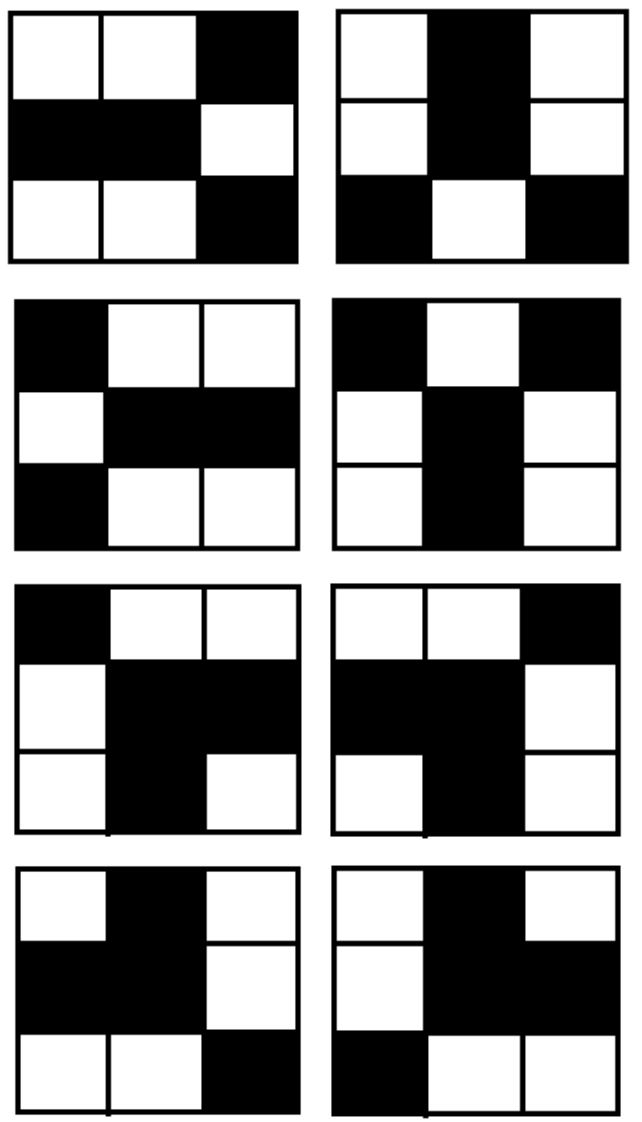

Fig. 5: The ridge bifurcation points represented in the form of pixels [22]

\subsection{Find the Minutiae Coordinates and Orientation Angles of a Thumbprint}

After converting the thinned (obtained by using inbuilt MATLAB function 'bwMorph()') image into black and white binary image it becomes ideal for the purpose of minutiae points extraction. The minutiae points are those points in the thumbrint from where the ridges bifurcate or converge. In order to find such minutiae points in the image we have written our own algorith $\mathrm{m}$ [22]. This algorith $\mathrm{m}$ uses a pattern searching technique in the binary image. The pattern to be searched is a two dimension binary pattern that forms a specific shape when viewed as a matrix. The pattern is a representation of a minutiae point which is present in the thumbprint in the form of a two dimensional matrix. The minutiae points are the bifurcation and the abrupt breakages of the ridges. The sample minutiae points that are present in an image will look like figure 4 . The ridge bifurcation patterns of figure 4 can be represented figure 5 . The patterns of figure 5 can be represented in the form of matrix of $3 \times 3$ dimensions by figure 6 .

As the image must be rotated in any direction, thus the pattern may also be present in many orientation and directions. Hence, to capture maximum possible patterns, we have to modify our matrices so as to include all kind of bifurcations and ridge breakages. After having many tests and observations we have found out that we can represent the ridge bifurcations in 24 different forms of matrices ( $\mathrm{m} 1$ to $\mathrm{m} 24)$, which are given figure 6. Similarly, we are able to represent the ridge breakages in 8 different forms of matrix patterns (m25 to $\mathrm{m} 32)$ as represented in figure 7 [22]:

\begin{tabular}{|l|l|l|}
\hline 0 & 0 & 1 \\
\hline 1 & 1 & 0 \\
\hline 0 & 0 & 1 \\
\hline
\end{tabular}

\begin{tabular}{|l|l|l|}
\hline 0 & 1 & 0 \\
\hline 0 & 1 & 0 \\
\hline 1 & 0 & 1 \\
\hline
\end{tabular}

\begin{tabular}{|l|l|l|}
\hline 1 & 0 & 0 \\
\hline 0 & 1 & 1 \\
\hline 1 & 0 & 0 \\
\hline
\end{tabular}

\begin{tabular}{|l|l|l|}
\hline 1 & 0 & 1 \\
\hline 0 & 1 & 0 \\
\hline 0 & 1 & 0 \\
\hline
\end{tabular}

\begin{tabular}{|l|l|l|}
\hline 1 & 0 & 0 \\
\hline 0 & 1 & 1 \\
\hline 0 & 1 & 0 \\
\hline
\end{tabular}

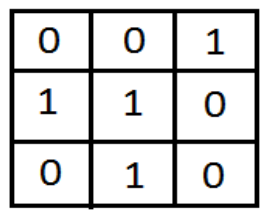

\begin{tabular}{|l|l|l|}
\hline 0 & 1 & 0 \\
\hline 1 & 1 & 0 \\
\hline 0 & 0 & 1 \\
\hline
\end{tabular}

\begin{tabular}{|l|l|l|}
\hline 0 & 1 & 0 \\
\hline 0 & 1 & 1 \\
\hline 1 & 0 & 0 \\
\hline
\end{tabular}

Fig. 6: Ridge bifurcations in integer format [22]

$$
\begin{aligned}
\mathrm{m} 1=[0,0,1 ; 1,1,0 ; 0,0,1] ; & \mathrm{m} 2=[0,1,0 ; 0,1,0 ; 1,0,1] ; \\
\mathrm{m} 3=[1,0,0 ; 0,1,1 ; 1,0,0] ; & \mathrm{m} 4=[1,0,1 ; 0,1,0 ; 0,1,0] ; \\
\mathrm{m} 5=[1,0,0 ; 0,1,1 ; 0,1,0] ; & \mathrm{m} 6=[0,0,1 ; 1,1,0 ; 0,1,0] ; \\
\mathrm{m} 7=[0,1,0 ; 1,1,0 ; 0,0,1] ; & \mathrm{m} 8=[0,1,0 ; 0,1,1 ; 1,0,0] ; \\
\mathrm{m} 9=[0,0,0 ; 1,1,1 ; 0,1,0] ; & \mathrm{m} 10=[0,1,0 ; 1,1,0 ; 0,1,0] ; \\
\mathrm{m} 11=[0,1,0 ; 1,1,1 ; 0,0,0] ; & \mathrm{m} 12=[0,1,0 ; 0,1,1 ; 0,1,0] \\
\mathrm{m} 13=[1,0,1 ; 0,1,0 ; 1,0,0] ; & \mathrm{m} 14=[1,0,1 ; 0,1,0 ; 0,0,1] ; \\
\mathrm{m} 15=[0,0,1 ; 0,1,0 ; 1,0,1] ; & \mathrm{m} 16=[1,0,0 ; 0,1,0 ; 1,0,1] ; \\
\mathrm{m} 17=[0,1,0 ; 1,1,1 ; 1,0,0] ; & \mathrm{m} 18=[0,1,0 ; 1,1,1 ; 0,0,1] ; \\
\mathrm{m} 19=[1,0,0 ; 1,1,1 ; 0,1,0] ; & \mathrm{m} 20=[0,0,1 ; 1,1,1 ; 0,1,0] ; \\
\mathrm{m} 21=[0,1,0 ; 0,1,1 ; 1,1,0] ; & \mathrm{m} 22=[1,1,0 ; 0,1,1 ; 0,1,0] ; \\
\mathrm{m} 23=[0,1,1 ; 1,1,0 ; 0,1,0] ; & \mathrm{m} 24=[0,1,0 ; 1,1,0 ; 0,1,1] ; \\
\mathrm{m} 25=[1,0,0 ; 0,1,0 ; 0,0,0] ; & \mathrm{m} 26=[0,1,0 ; 0,1,0 ; 0,0,0] \\
\mathrm{m} 27=[0,0,1 ; 0,1,0 ; 0,0,0] ; & \mathrm{m} 28=[0,0,0 ; 0,1,1 ; 0,0,0] \\
\mathrm{m} 29=[0,0,0 ; 0,1,0 ; 0,0,1] ; & \mathrm{m} 30=[0,0,0 ; 0,1,0 ; 0,1,0] \\
\mathrm{m} 31=[0,0,0 ; 0,1,0 ; 1,0,0] ; & \mathrm{m} 32=[0,0,0 ; 1,1,0 ; 0,0,0]
\end{aligned}
$$

Fig. 7: Representation of ridge bifurcation and ridge breakage in matrix format [22] 
To detect these patterns in the thumb print image, we perform some pre-processing steps after which we get the thinned ridge image. We have to search these $3 \times 3$ dimensional images in the thinned ridge image using simple pattern searching.

Each minutia point will have $\mathrm{X}$ coordinate, $\mathrm{Y}$ coordinate, and orientation angle $\theta$. The steps for finding minutiae coordinates and orientation angles of a thumbprint image are presented figure 8 .

\subsection{Matching Results}

Find the union of minutiae coordinates and orientation angles of first $\mathrm{n}$ thumbprints for a person using Eq. (5) and store these values in the memory for further identification. Here, values of $\mathrm{n}$ may vary from two to six. The stored minutiae values will be compared with minutiae coordinates and orientation angles of an online scanned thumbprint. If online calculated minutiae values have $80 \%$ and above matching score then it means that the stored and online calculated minutiae values are belonging to the same person.

Step 1: Read the thumbprint image of identical twin using MATLAB 7.0 predefined function. $i=$ imread("imagename");

Step 2: Convert the image into binary image using predefined function.

$$
j=i m 2 b w(i) \text {; }
$$

Step 3: Convert the gray scale image into binary image using predefined function.

$$
i b w=i m 2 b w(j) \text {; }
$$

Step 4: Thinning the ridges of thumbprint using predefined function.

thin_image $=$ bwmorph('ibw', 'thin', 'inf'),

Step 5: Use our own function getminutiaes( ) for finding the minutiae points.

list_of_minutiae_points = getminutiae (thinned_image);

Step 6: Find the orientation angles using our own function showdirection( ).

orientation_angles $=$ showdirection(thinned_image);

Fig. 8: The steps of an algorithm for finding minutiae coordinates and orientation angles of a thumbprint $[22,23,24]$

\section{Experimental Analysis and Results}

\subsection{Analysis of Results for Actual Dataset}

The proposed method is tested on self generated identical twin dataset using $\mathrm{H} 3 \mathrm{~T}$ \& A terminal and

FVC datasets. For each pers on of the identical twin pair nine thumbprint images were scanned. The actual size thumbprints of identical twin pair (Servesh and Dhermesh) are varying from S1 to S9 and D1 to D9 in figure 9.

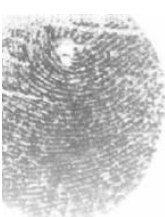

9(a) S1

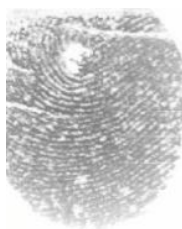

9(c) S2

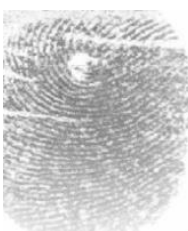

9(e) S3

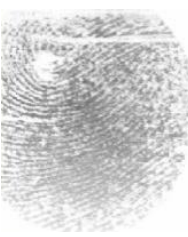

9(g) S4

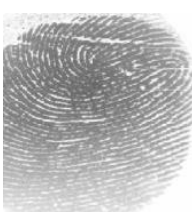

9(i) $\mathrm{S} 5$

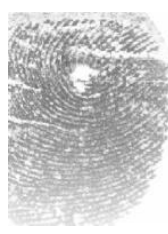

9(k) S6

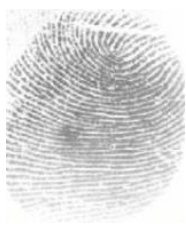

9(b) D1

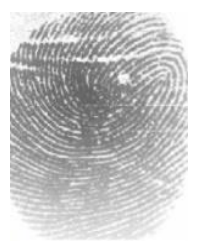

9(d) D2

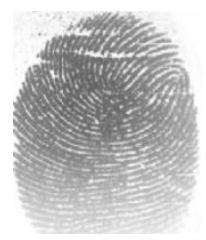

9(f) D3

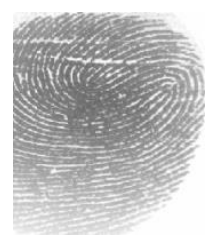

9(h) D4

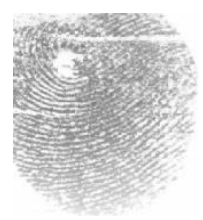

9(j) D5

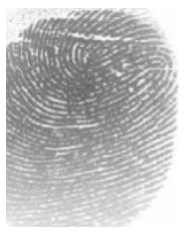

9(1) D6 


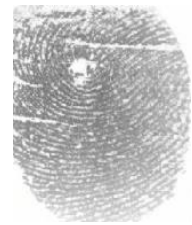

$9(\mathrm{~m}) \mathrm{S} 7$

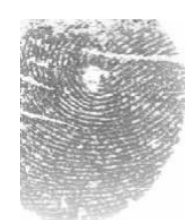

9(o) S8

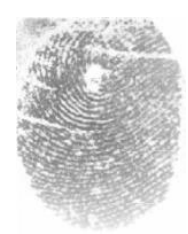

9(q) S9

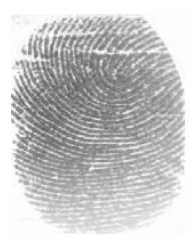

9(n) D7

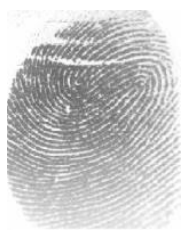

$9(\mathrm{p}) \mathrm{D} 8$

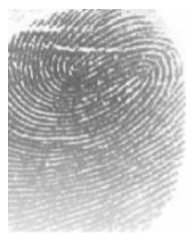

9(r) D9
Fig. 9: Thumbprint images of Servesh(S) and Dhermesh(D)

The thinned images of figure 10 are obtained after implementing steps 1 to 6 of section IV. The thinned images for thumbprints of figure 9 in actual format are represented by figure 10 .

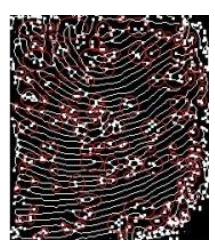

10(a) S1

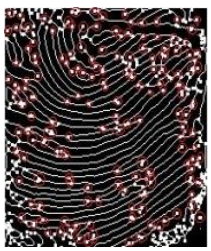

10(c) S2

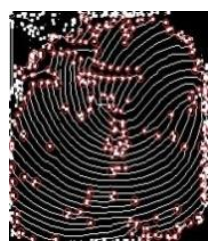

$10(\mathrm{e}) \mathrm{S} 3$

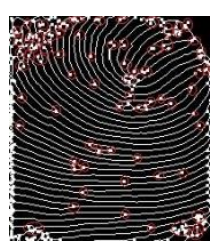

10(b) D1

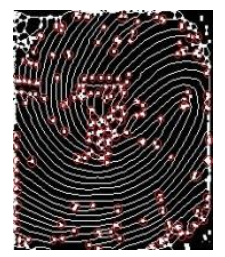

10(d) D2

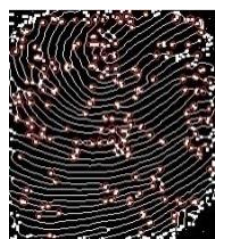

10(f) D3

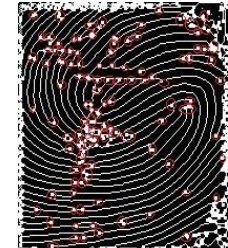

$10(\mathrm{~g}) \mathrm{S} 4$

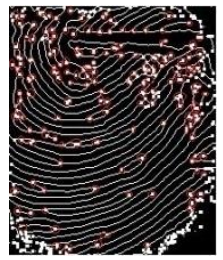

10(i) S5

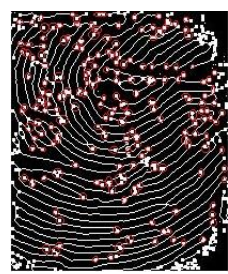

10(k) S6

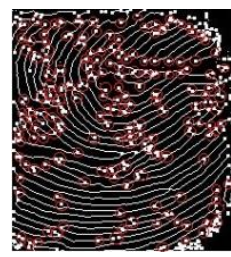

$10(\mathrm{~m}) \mathrm{S} 7$

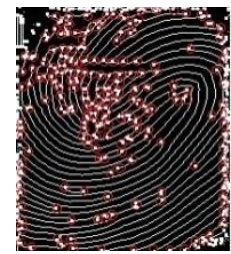

10(0) S8

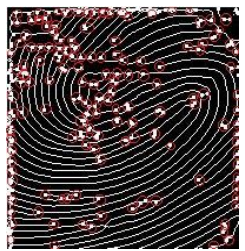

10(q) $\mathrm{S} 9$

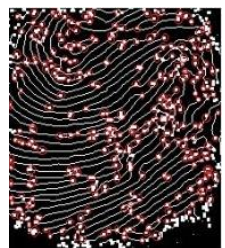

10(h) D4

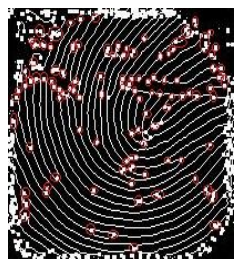

10(j) D5

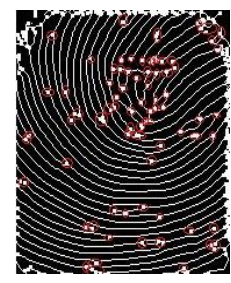

10(1) D6

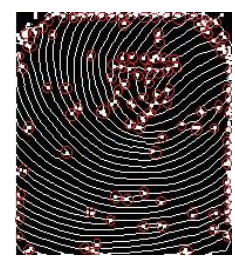

10(n) D7

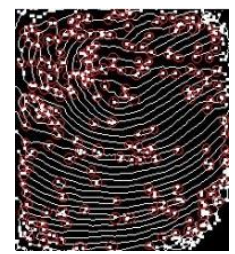

10(p) D8

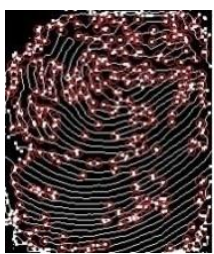

10(r) D9
Fig. 10: Nine pairs of thinned thumbprint images of Servesh(S) and Dhermesh(D) [23, 24] 


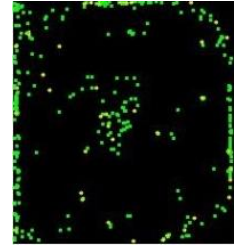

11(a) S1

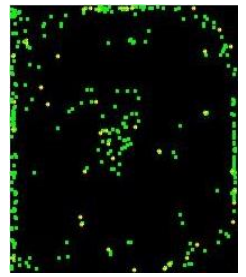

$11(\mathrm{c}) \mathrm{S} 2$

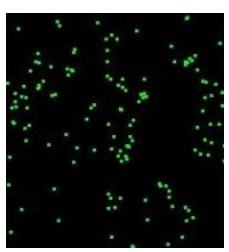

11(e) S3

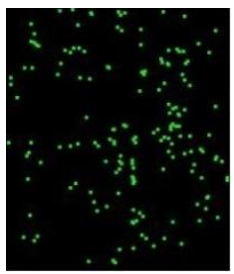

$11(\mathrm{~g}) \mathrm{S} 4$

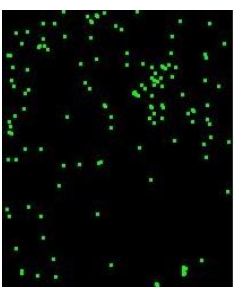

11(i) S5

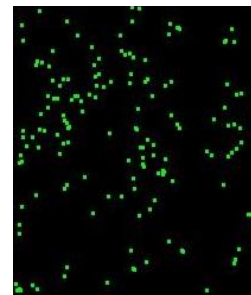

$11(\mathrm{k}) \mathrm{S} 6$

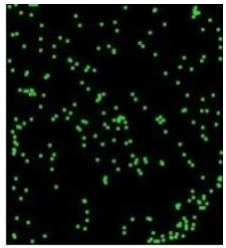

11(b) D1

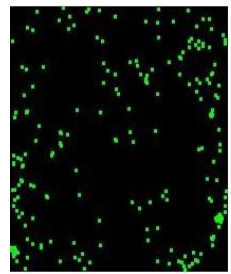

11(d) D2

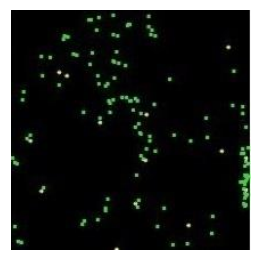

11(f) D3

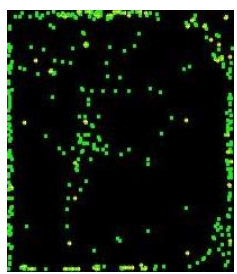

11(h) D4

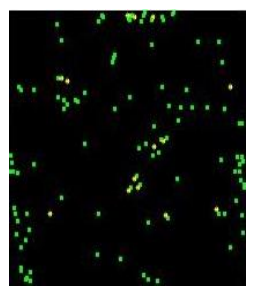

11(j) D5

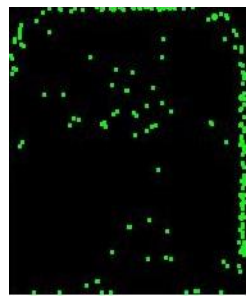

11(1) D6
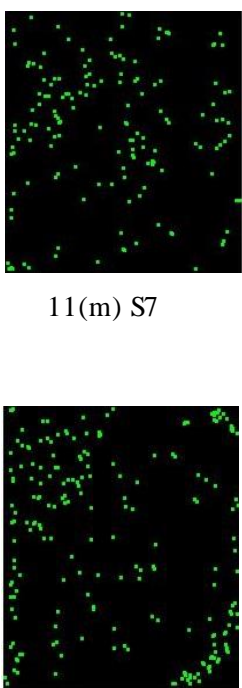

11(o) S8

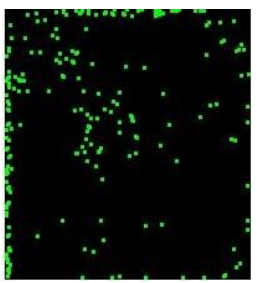

11(q) S9 11(m) S7

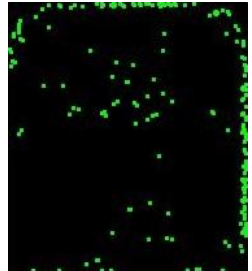

11(n) D7

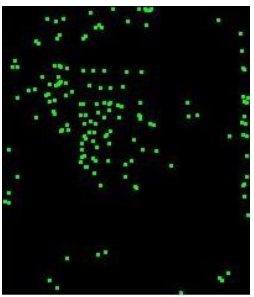

11(p) D8

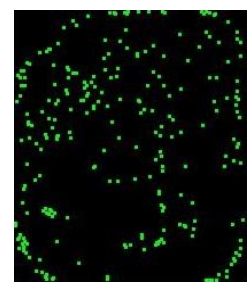

11(r) D9
Fig. 11: Nine pairs of minutiae coordinates and orientation angles for thumbprint images of Servesh(S) and Dhermesh(D) [23, 24]

In figure 10 , the minutiae points are circled. The minutiae orientation angles of each thinned image of figure 10 are obtained after applying steps 5 and 6 of the proposed algorithm of section IV.B. The orientation angles for thumbrints of figure 10 are represented by figure 11.

The union set for each person of identical twin is obtained after combining the minutiae coordinates and orientation angles of first six thumbprint images of Servesh (S1, S2, S3, S4, S5, S6) and Dhermesh (D1, D2, D3, D4, D5, D6). The thumbprints from S7 to S9 and D7 to D9 of figure 11 are used for comparing matching results.

As per figure 11, the minutiae union set of Servesh has approximately 750 to 800 minutiae coordinates with orientation angles. The minutiae union set of Dhermesh has approximately 850 to 900 minutiae coordinates and orientation angles. The union sets of Servesh and Dhermesh are used for online comparison and identification of thumbprints. If a single coordinate $(\mathrm{X}, \mathrm{Y}, \theta)$ is stored as an independent integer number in the memory then it will take four bytes of memory. Therefore, on the basis of results of figures 11 we can conclude that the minutiae union set of Servesh can be stored in $2.5 \mathrm{~KB}$ to $3 \mathrm{~KB}$ memory and the same of Dhermesh will require $3 \mathrm{~KB}$ to $3.5 \mathrm{~KB}$ memory 
On the basis of $7^{\text {th }}, 8^{\text {th }}$, and $9^{\text {th }}$ thumbprints of figure 11 , we can conclude that approximately 150 to 170 minutiae points are there in a thumbprint of Dhermesh. If we store a thu mbprint of Servesh or Dhermesh in the form of minutiae coordinates then we need approximately 600bytes to 800 bytes of memory. The result comparisons of Servesh and Dhermesh thumbprints with their union minutiae sets are presented in tables 1,2 , and 3 .

Table 1: Comparing results of Servesh thumbprints union set with 7 th, $8^{\text {th }}$, and $9^{\text {th }}$ thumbprints of Servesh

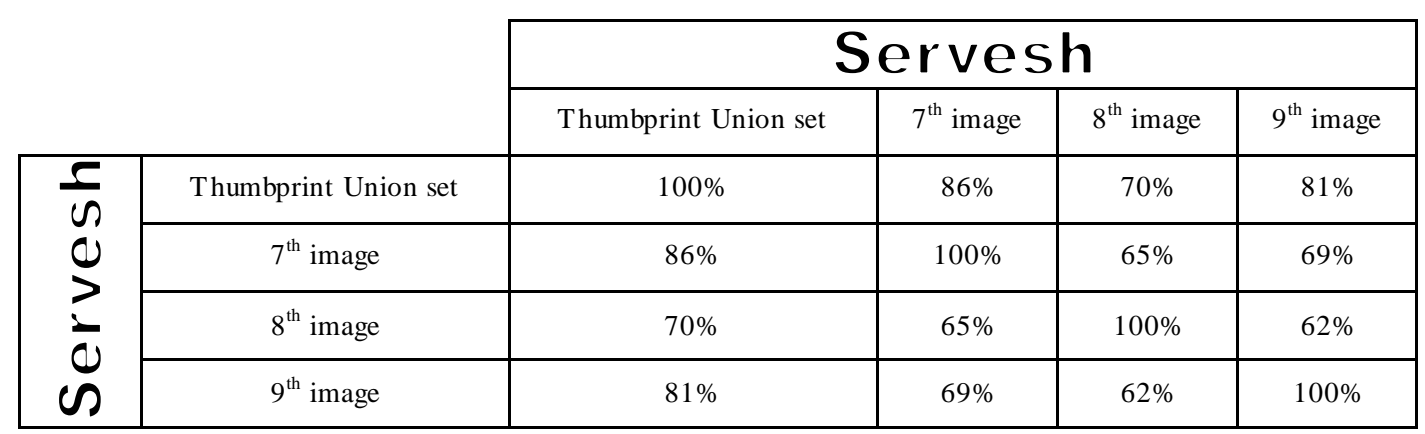

Table 2: Comparing results of Dhermesh thumbprints union set with 7 th, $8^{\text {th }}$, and $9^{\text {th }}$ thumbprints of Dhermesh

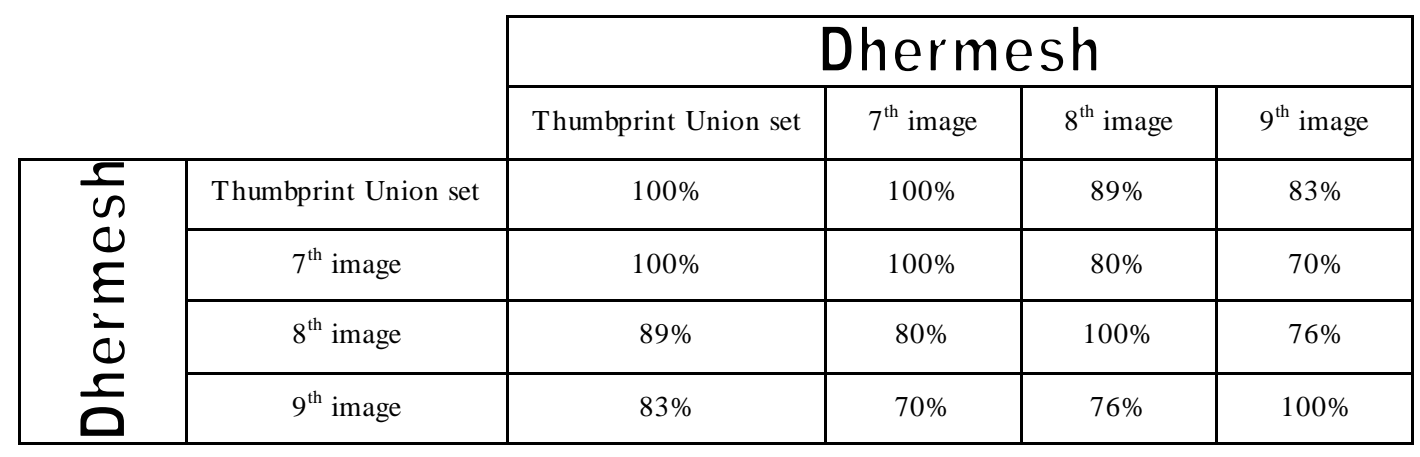

Table 3: Comparing the results of thumbprints of Servesh and Dhermesh

\begin{tabular}{|c|c|c|c|c|c|}
\hline & \multicolumn{4}{|c|}{ Dhermesh } \\
\hline & & Thumbprint Union set & $7^{\text {th }}$ image & $8^{\text {th }}$ image & $9^{\text {th }}$ image \\
\hline \multirow{4}{*}{$\begin{array}{l}\frac{c}{u} \\
0 \\
0 \\
0 \\
0\end{array}$} & Thumbprint Union set & & $25.2 \%$ & $26 \%$ & $60 \%$ \\
\hline & $7^{\text {th }}$ image & $32.3 \%$ & $15 \%$ & $14 \%$ & $23 \%$ \\
\hline & $8^{\text {th }}$ image & $33 \%$ & $22 \%$ & $25 \%$ & $27 \%$ \\
\hline & $9^{\text {th }}$ image & $30 \%$ & $12 \%$ & $20 \%$ & $39 \%$ \\
\hline
\end{tabular}

The results of table 1 show that a new thumbprint of Servesh is very close to the union set of Servesh and the results of table 2 show that a new thumbprint of Dhermesh is very similar to that of Dhermesh minutiae union set.

The comparisons of new thumbprints of Servesh and Dhermesh with each other are presented in table 3 . The results of table 3 show that minutiae coordinates with orientation angles of Servesh and Dhermesh thumbprints are totally different. Therefore, Dhermesh and Servesh can be differentiated and identified on the basis of their thumbrints minutiae union sets.

\subsection{Analysis of Results for FVC Datasets}

The proposed minutiae fusion based method presented in section 4 was tested for FVC2004 and FVC2006 standard datasets. The FVC datasets have more than 100 identical twins thumbprints. 


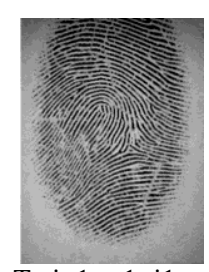

Twin1_p1_i1

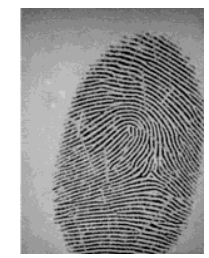

Twin1_p1_i2

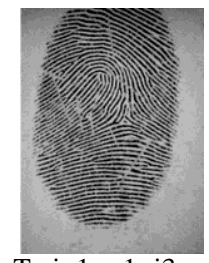

Twin1_p1_i3

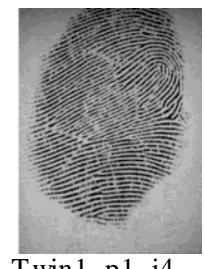

Twin1_p1_i4
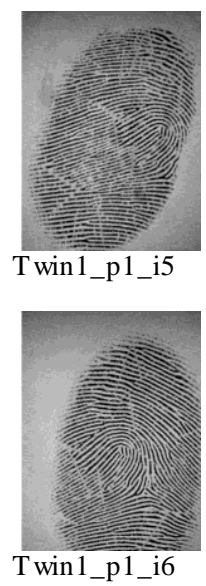
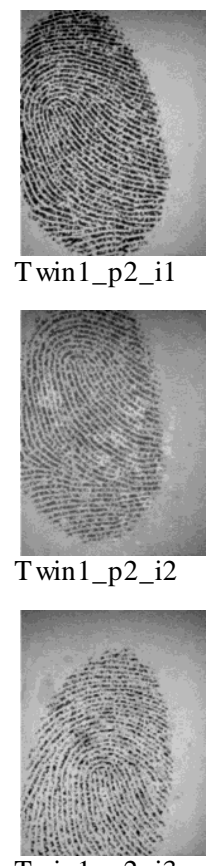

Twin1_p2_i3
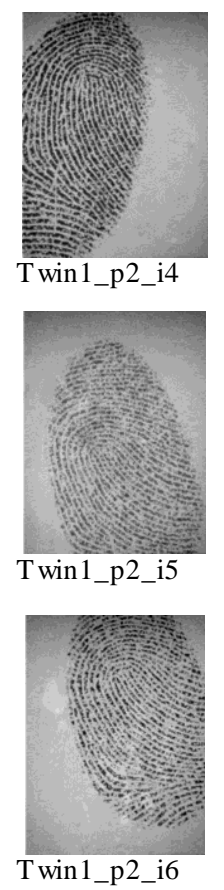

Fig. 12: First twin pair thumbprint images for FVC datasets [8, 9]

For each person eight thumbprints are provided by FVC dataset but only six thumbprints are of good quality. Therefore, we have considered these six thumbprints of each person for analyzing the performance of our proposed algorithm. The thumbprints of two different pairs of identical twins for FVC datasets are presented in figures 12 and 13 respectively.
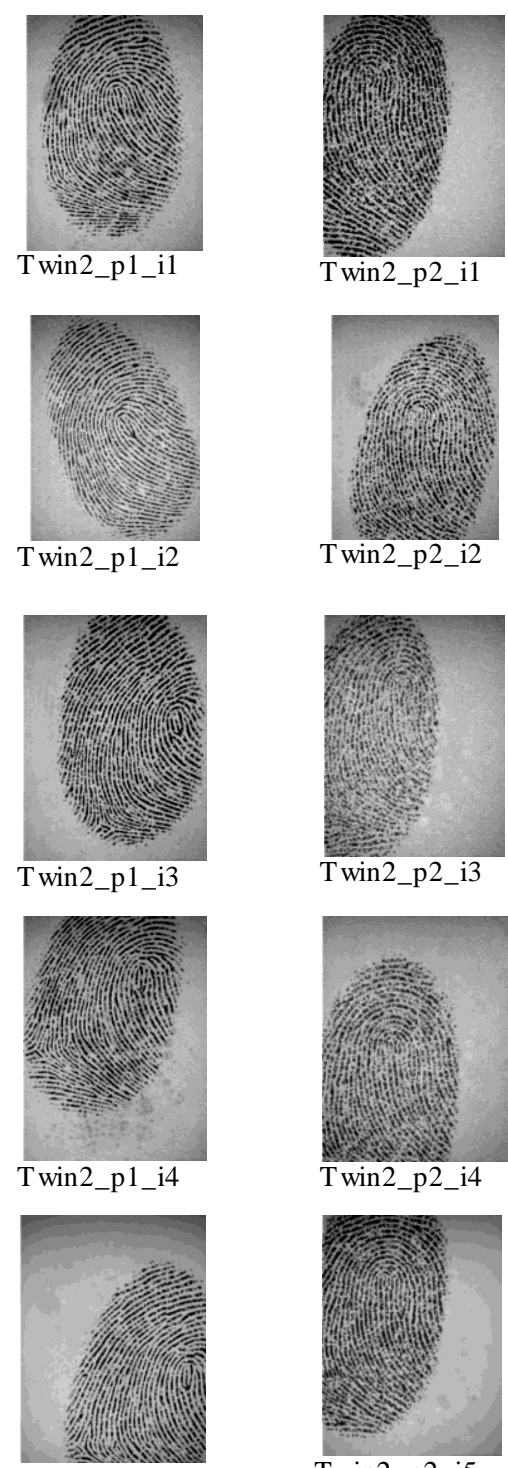

Twin2_p1_i5

Twin2_p2_i5
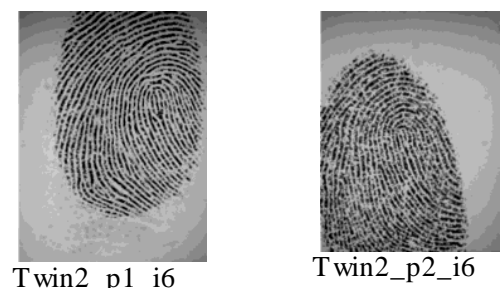

Fig. 13: Second twin pairs thumbprint images for FVC dat asets $[8,9]$

We implemented the steps of section IV on first four thumbprints for a person of identical twin pairs specified in figures 12 , and 13 . The union of minutiae coordinates and orientation angles of first four thumbprints of a person for FVC dataset were considered as minutiae union set. The fifth and sixth images of figures 12 and 13 were compared with minutiae fuzzy sets for identity verification.

For comparing a thumbprint image with minutiae union sets we deviated the minutiae coordinates from 10 to 10 and the best comparison results were taken 
into considerations. The result comparisons of $5^{\text {th }}$ and $6^{\text {th }}$ thumbprints of identical twins with minutiae union sets for FVC2004 and FVC2006 datasets are presented in table 4 .

Table 4: Comparing the results of FVC04 and FVC06 dataset thumbprints

\begin{tabular}{|c|c|c|c|c|}
\cline { 2 - 5 } & \multicolumn{4}{|c|}{ FVC4 and FVC6 data set } \\
\cline { 2 - 5 } & $\begin{array}{c}\text { Twin1_P1 Thumb } \\
\text { Print Fusion }\end{array}$ & $\begin{array}{c}\text { Twin1_P2 Thumb } \\
\text { Print Fusion }\end{array}$ & $\begin{array}{c}\text { Twin2_P1 Thumb Print } \\
\text { Fusion }\end{array}$ & $\begin{array}{c}\text { Twin2_P2 Thumb Print } \\
\text { Fusion }\end{array}$ \\
\hline Twin1_p1_5 & $78 \%$ & $22 \%$ & $10 \%$ & $16 \%$ \\
\hline Twin1_p1_6 & $82 \%$ & $20 \%$ & $21 \%$ & $19 \%$ \\
\hline Twin1_p2_5 & $14 \%$ & $80 \%$ & $10 \%$ & $13 \%$ \\
\hline Twin1_p2_6 & $18 \%$ & $79 \%$ & $11 \%$ & $12 \%$ \\
\hline Twin2_p1_5 & $10 \%$ & $18 \%$ & $81 \%$ & $9 \%$ \\
\hline Twin2_p1_6 & $11 \%$ & $13 \%$ & $16 \%$ \\
\hline Twin2_p2_5 & $13 \%$ & $16 \%$ & $18 \%$ & $77 \%$ \\
\hline Twin2_p2_6 & $8 \%$ & $9 \%$ & & $81 \%$ \\
\hline
\end{tabular}

Table 5: Memory requirements for actual size thumbprints, minutiae fuzzy sets, and an independent minutiae thumb

\begin{tabular}{|c|c|c|c|c|}
\cline { 2 - 5 } \multicolumn{1}{|c|}{} & Person & $\begin{array}{c}\text { Actual size of thumbprint } \\
\text { (in KB) }\end{array}$ & $\begin{array}{c}\text { Minutiae thumb } \\
\text { Union set memory size } \\
\text { (in KB) }\end{array}$ & $\begin{array}{c}\text { An independent minutiae thumb size } \\
\text { (in KB) }\end{array}$ \\
\hline 1. & Servesh & $55 \mathrm{~KB}$ to $60 \mathrm{~KB}$ & $2.5 \mathrm{~KB}$ to $3 \mathrm{~KB}$ & $0.7 \mathrm{~KB}$ to $0.8 \mathrm{~KB}$ \\
\hline 2. & Dhermesh & $55 \mathrm{~KB}$ to $60 \mathrm{~KB}$ & $3.0 \mathrm{~KB}$ to $3.5 \mathrm{~KB}$ & $0.8 \mathrm{~KB}$ to $0.9 \mathrm{~KB}$ \\
\hline 3. & FVC_twin1_p1 & $105 \mathrm{~KB}$ to $110 \mathrm{~KB}$ & $3.0 \mathrm{~KB}$ to $4.0 \mathrm{~KB}$ & $0.8 \mathrm{~KB}$ to $1.0 \mathrm{~KB}$ \\
\hline 4. & FVC_twin1_p2 & $105 \mathrm{~KB}$ to $110 \mathrm{~KB}$ & $3.0 \mathrm{~KB}$ to $4.0 \mathrm{~KB}$ & $0.8 \mathrm{~KB}$ to $1.0 \mathrm{~KB}$ \\
\hline 5. & FVC_twin2_p1 & $105 \mathrm{~KB}$ to $110 \mathrm{~KB}$ & $3.0 \mathrm{~KB}$ to $4.0 \mathrm{~KB}$ & $0.8 \mathrm{~KB}$ to $1.0 \mathrm{~KB}$ \\
\hline 6. & FVC_twin2_p2 & $105 \mathrm{~KB}$ to $110 \mathrm{~KB}$ & $3.0 \mathrm{~KB}$ to $4.0 \mathrm{~KB}$ & $0.8 \mathrm{~KB}$ to $1.0 \mathrm{~KB}$ \\
\hline
\end{tabular}

The results of table 4 show that the $5^{\text {th }}$ and $6^{\text {th }}$ thumbprints are very close to their corresponding minutiae union sets. After comparing twin1_p1_5 $5^{\text {th }}$ and twin1_p1_6 $6^{\text {th }}$ images with twin1_p2_thumbprint, twin2_p1_thumbprint and twin2_p2_thumbprint union sets, we observed that identical twins thumbprints are totally different in terms of minutiae coordinates and orientation angles values.

Similar analysis was conducted for fifty identical twins of FVC thumbprint datasets and we observed that the identical twins of FVC thumbprint datasets are dissimilar with each other.

The memory requirements for minutiae union sets of standard and self generated data sets are given in table 5. The actual size of Servesh and Dhermesh thumbprints are in the range of $55 \mathrm{~KB}$ to $60 \mathrm{~KB}$. The actual memory requirements for FVC thumbprint datasets are varying from $105 \mathrm{~KB}$ to $110 \mathrm{~KB}$. The minutiae thumb union sets for Servesh and Dhermesh require approximately $3.0 \mathrm{~KB}$ memory. If we store an independent thumbprint image in the integer form of minutiae coordinates and orientation angles then we need approximately $0.7 \mathrm{~KB}$ to $1.0 \mathrm{~KB}$ memory for self generated and standard datasets.

\subsection{Result Analysis for Fusion of Two and More Thumbprints of Identical Twins}

We took the union sets of minutiae coord inates and orientation angles for two to six thumbprints of identical twins and compared with randomly selected thumbprints. These results are presented in figure 14 .

The results of figure 14 , show that if we compare the fusion of two thumbrints of Servesh with a randomly selected thumbprint of Servesh then the match percentage is approximately $80 \%$ whereas if we compare the fusion of two thumbprints of Servesh with randomly selected thumbprint of Dhermesh then the match percentage is bellow $20 \%$. The thumbprint matching percentage for the same person increases if we increase the number of thumbprints of union set. 


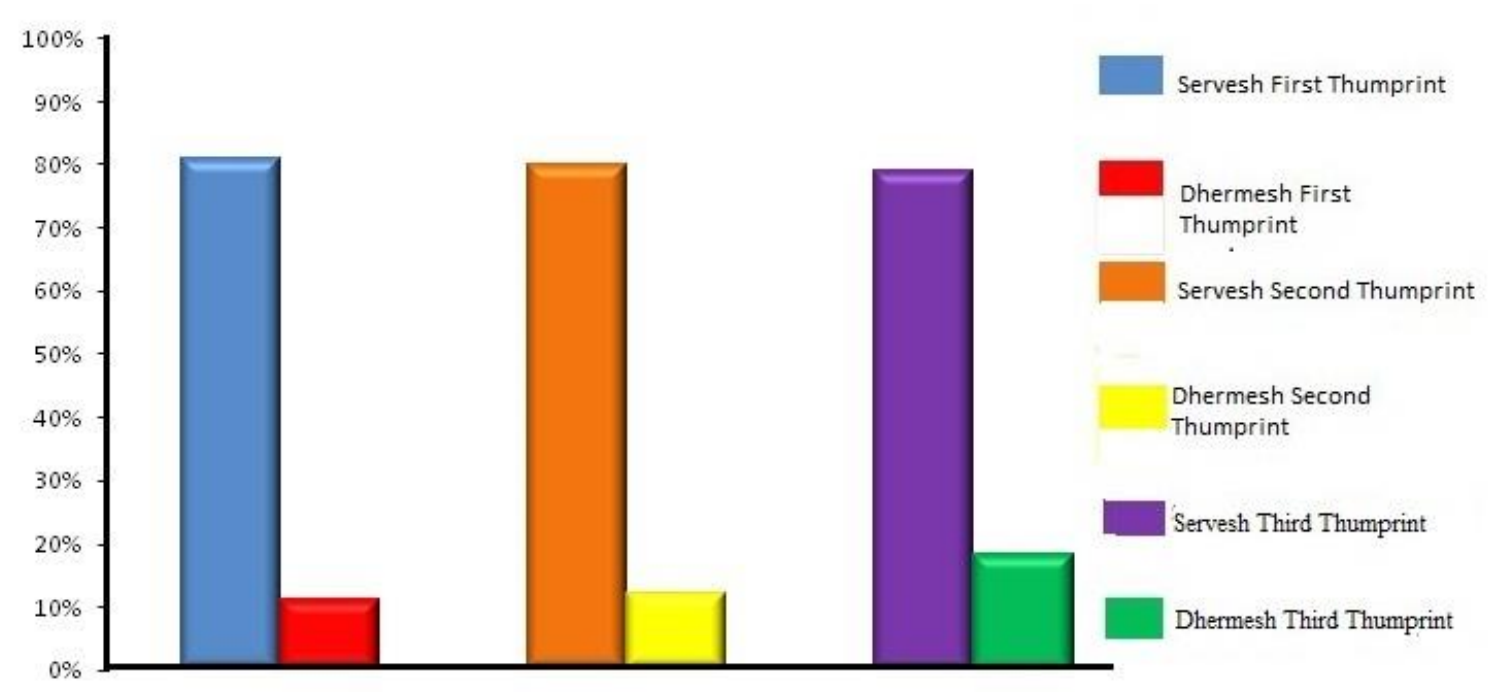

Fig. 14(a): Comparing the fusion of two thumbprints of Servesh with randomly selected th umbprints of Servesh \& Dhermesh

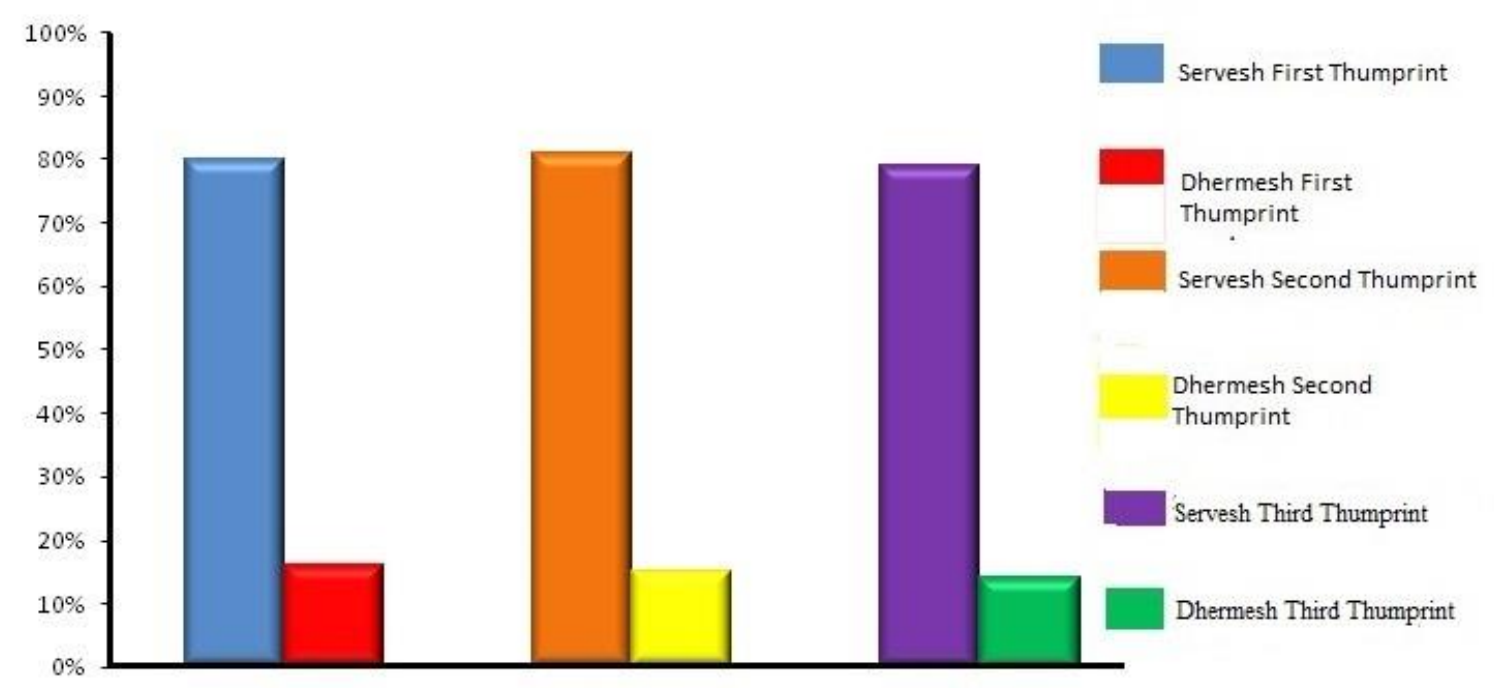

Fig. 14(b): Comparing the fusion of three thumbprints of Servesh with randomly selected thumbprints of Servesh \& Dhermesh

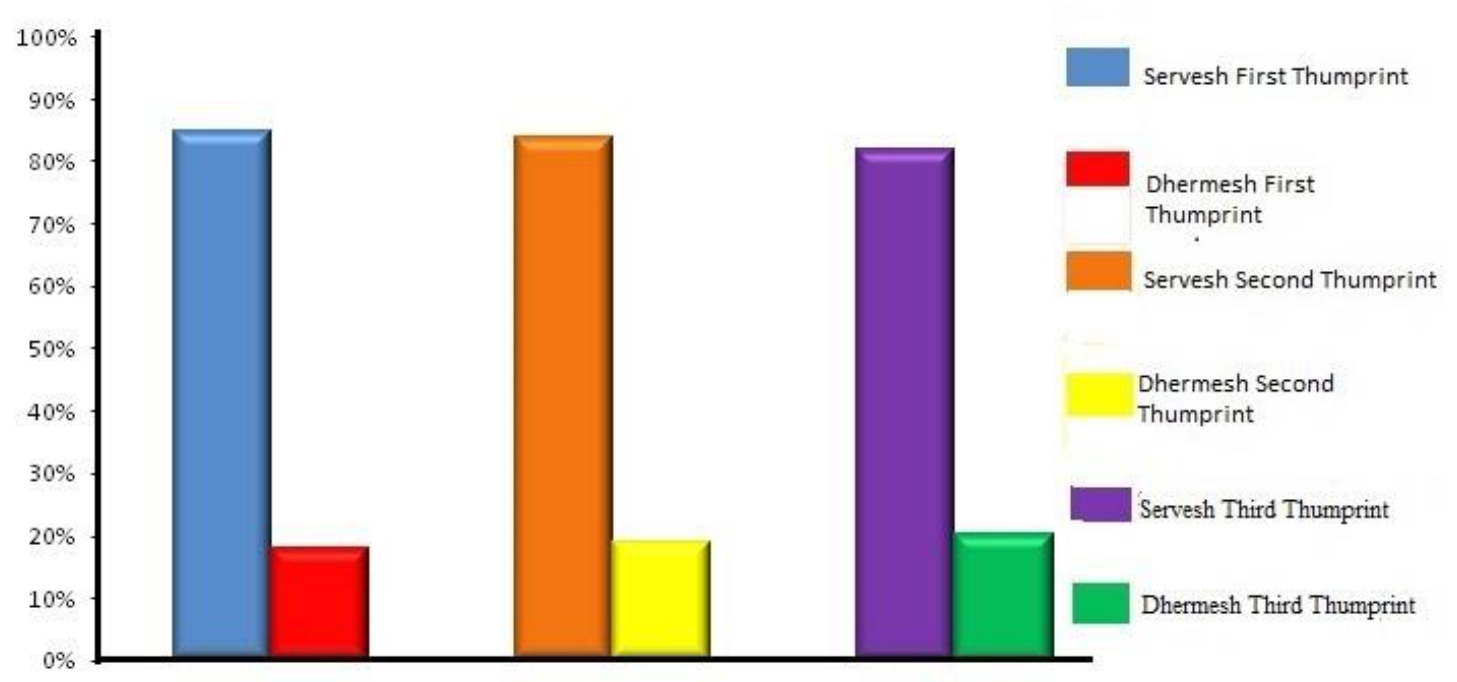

Fig. 14(c) Comparing the fusion of four thumbprints of Servesh with randomly selected thumbprints of Servesh \& Dhermesh. 


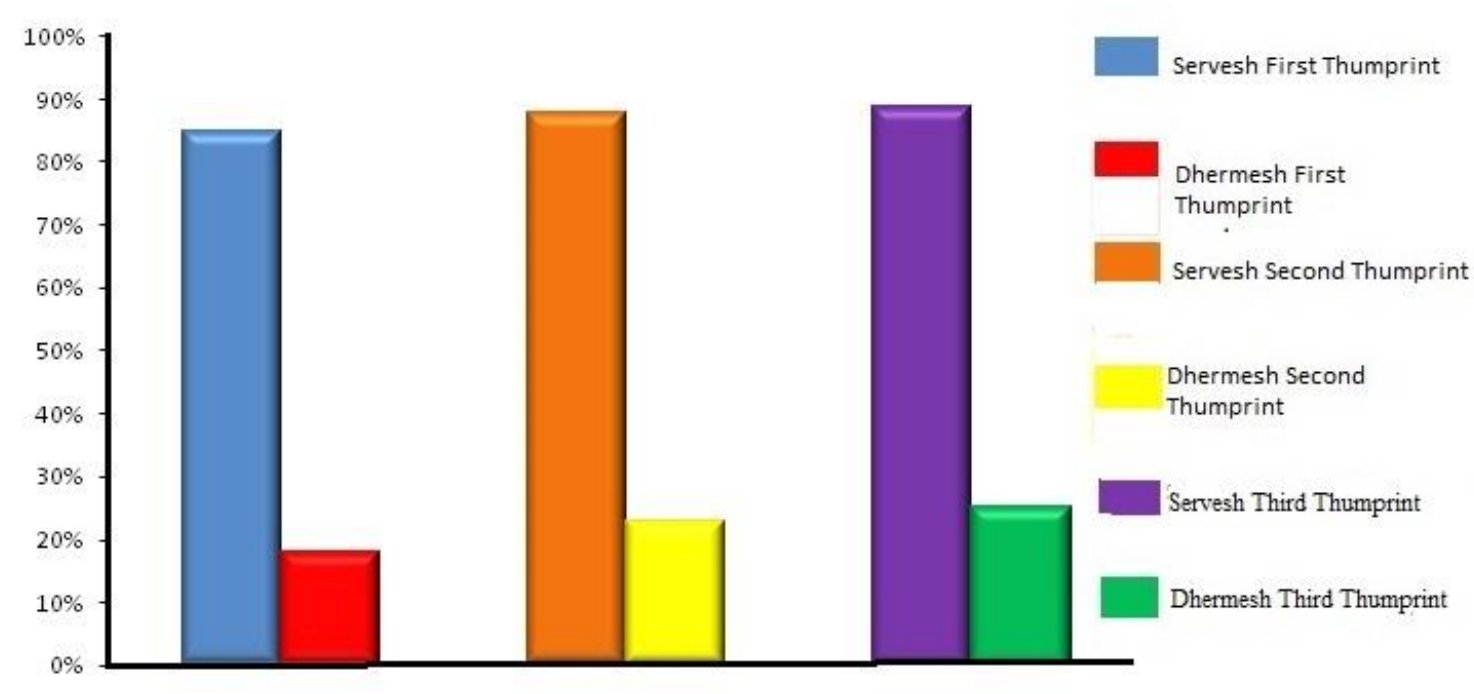

Fig. 14(d): Comparing the fusion of five thumbprints of Servesh with randomly selected thumbprints of Servesh \& Dhermesh

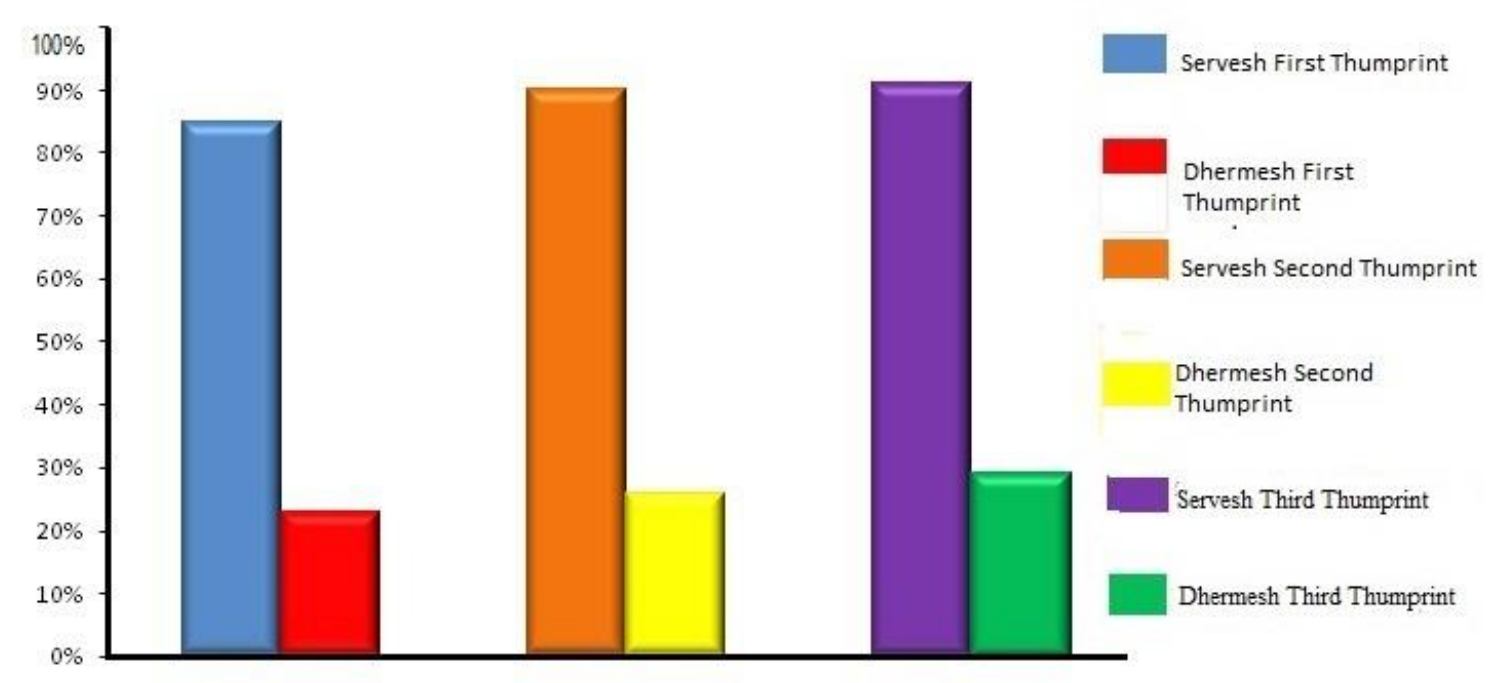

Fig. 14(e): Comparing the fusion of six thumbprints of Servesh with randomly selected thumbprints of Servesh \& Dhermesh.

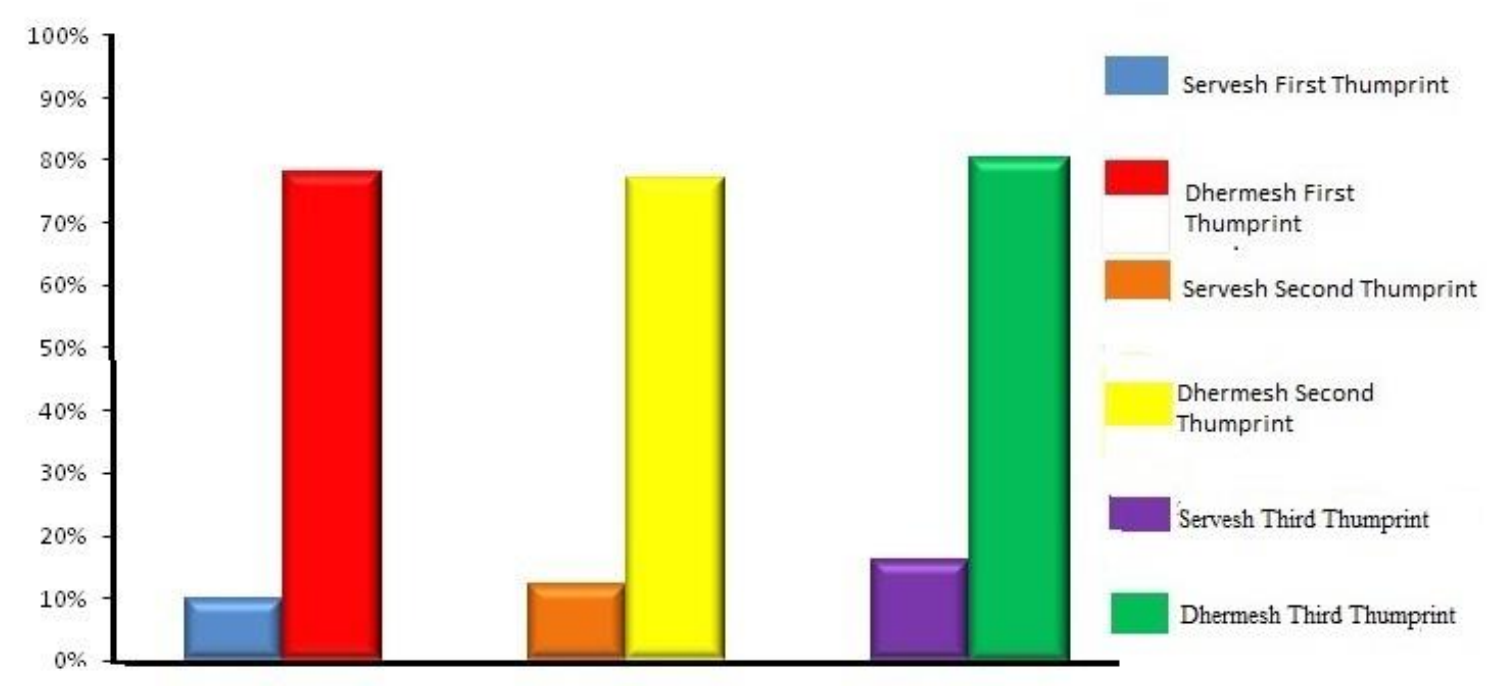

Fig. 14(f): Comparing the fusion of two thumbprints of Dhermesh with randomly selected thumbprints of Dhermesh \& Servesh 


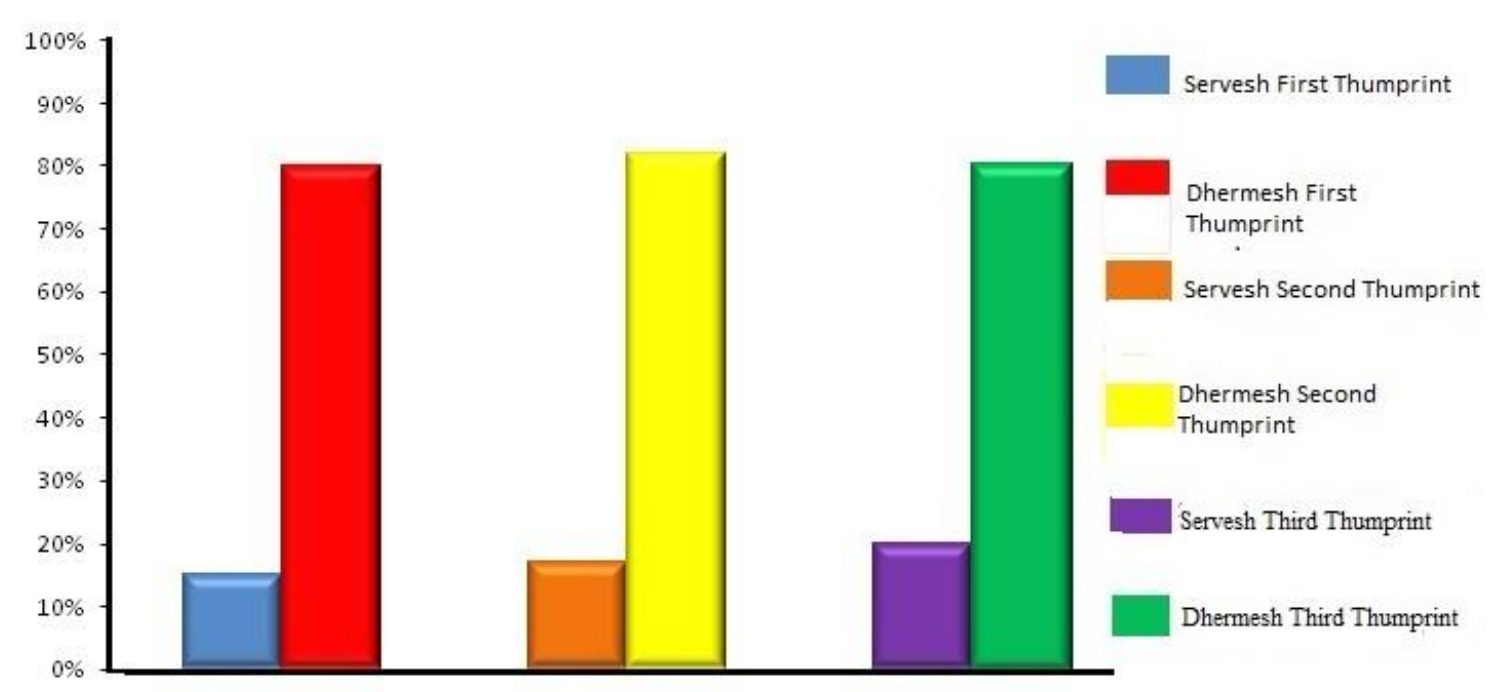

Fig. 14(g): Comparing the fusion of three thumbprints of Dhermesh with randomly selected thumbprints of Dhermesh \& Servesh.

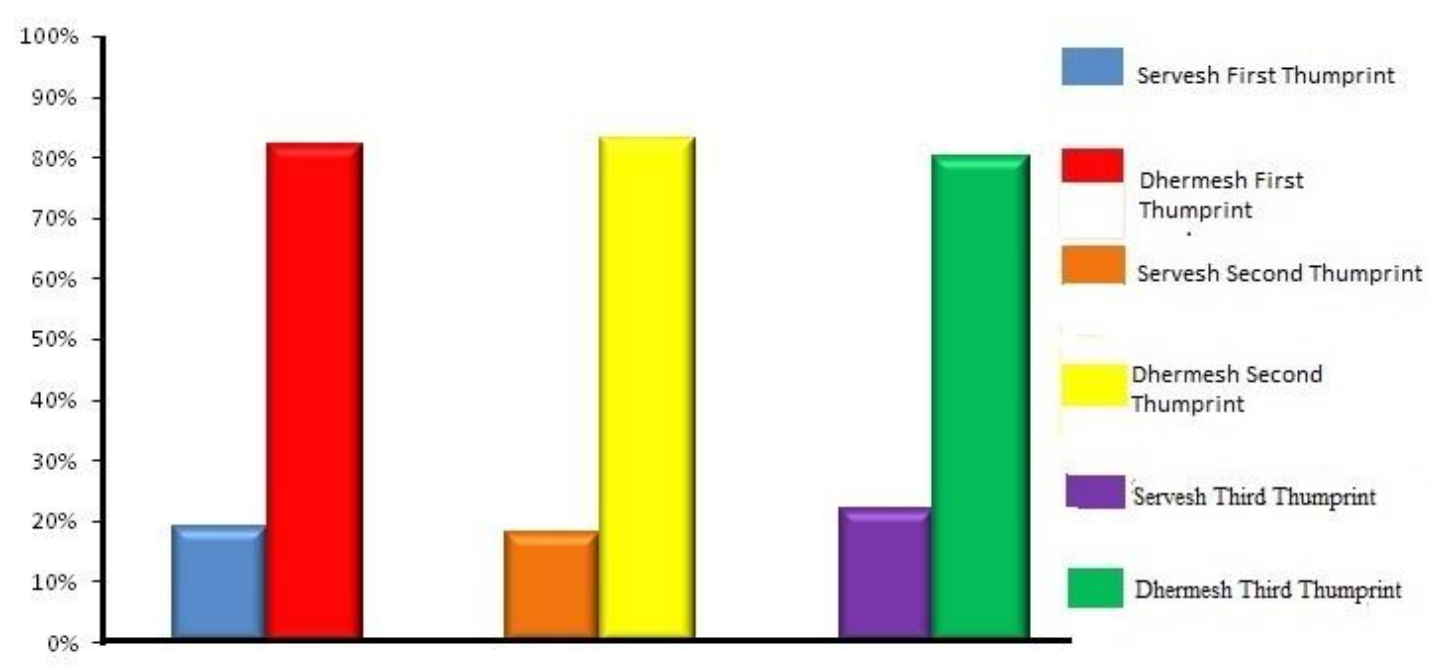

Fig. 14(h): Comparing the fusion of four thumbprints of Dhermesh with randomly selected thumbprints of Dhermesh \& Servesh.

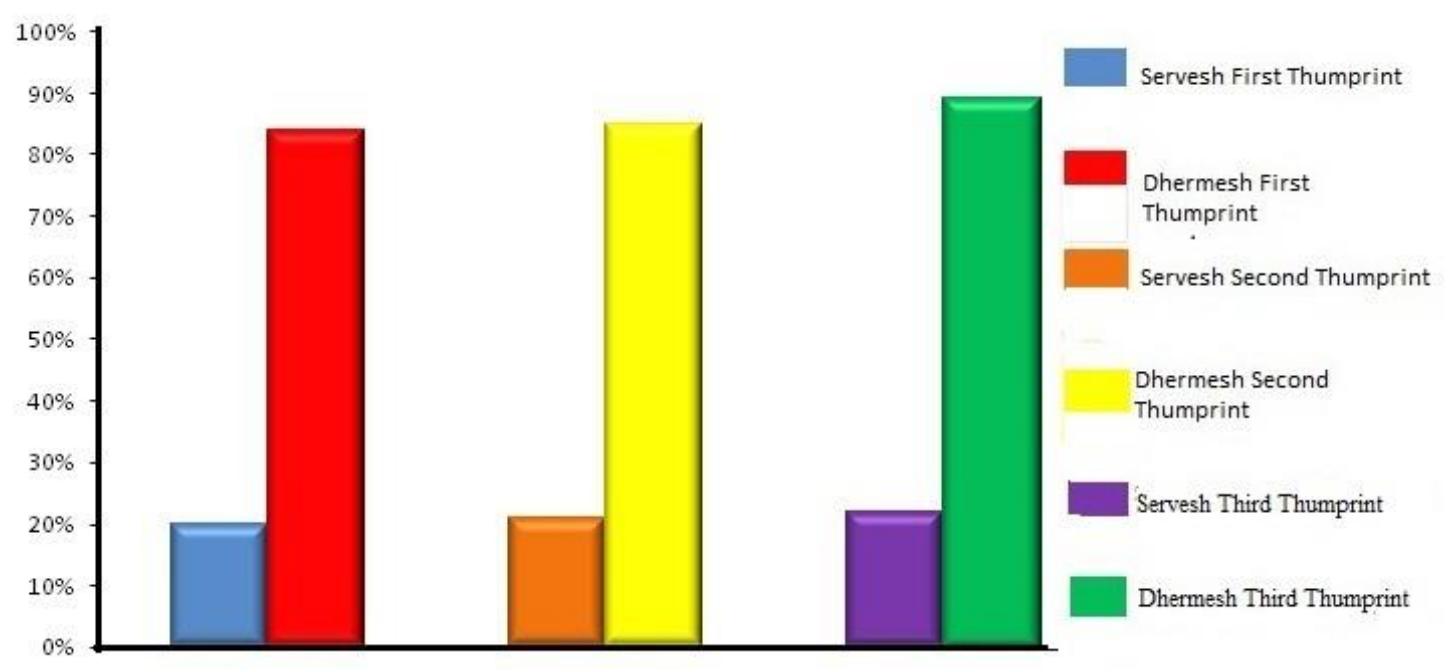

Fig. 14(i): Comparing the fusion of five thumbprints of Dhermesh with randomly selected thumbprints of Dhermesh \& Servesh. 


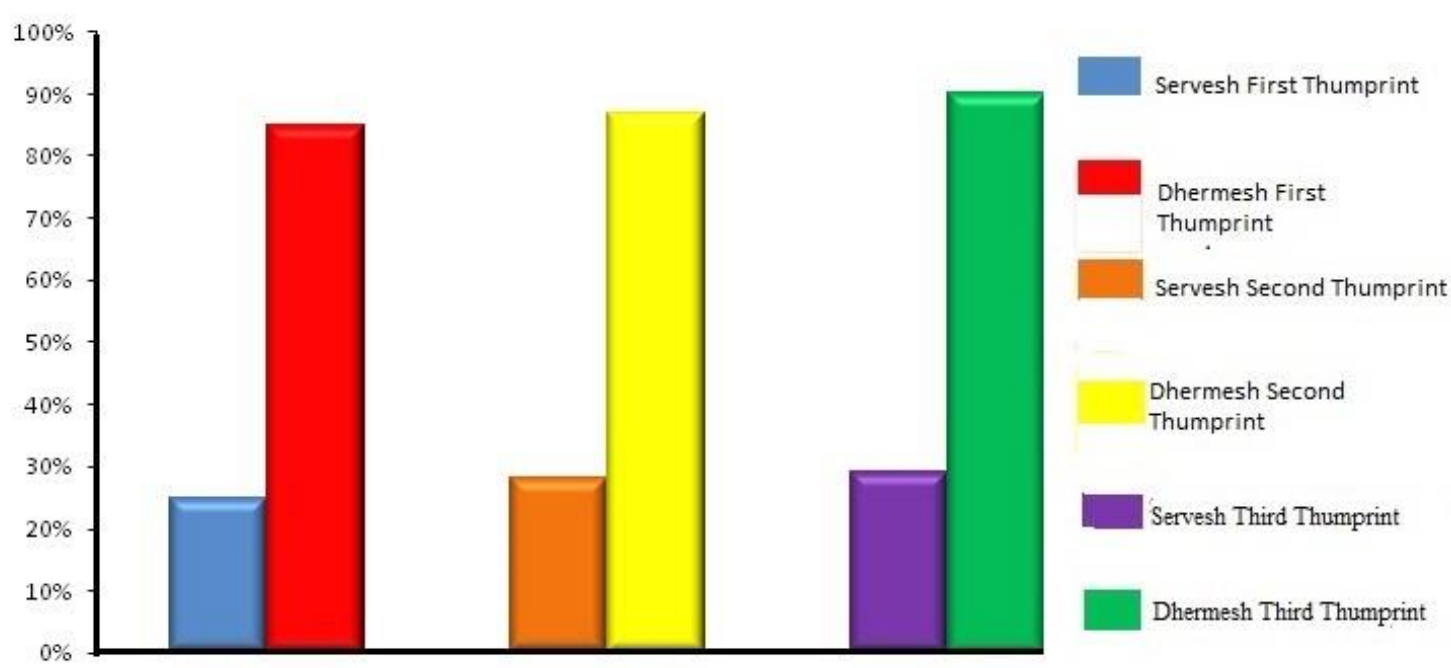

Fig. 14(j): Comparing the fusion of two thumbprints of Dhermesh with randomly selected thumbprints of Servesh and Dhermesh

Fig. 14: Comparing the fusion of two to six thumbprints of identical twins with randomly selected thumbprints

Table 6: Comparing the results of proposed method with other methods.

\begin{tabular}{|c|c|c|c|}
\cline { 2 - 4 } \multicolumn{1}{c|}{} & Algorithm & Matcher Name & Matching Results (Identical Twins) \\
\hline $\mathbf{1 .}$ & Jain [13] & Minutiae & FAR: 2 - $6 \%$ higher \\
\hline $\mathbf{2 .}$ & Han [6] & Minutiae & EER: $1-2 \%$ higher \\
\hline $\mathbf{3 .}$ & Srihari [27] & NFIS & EER: $6.17 \%$ \\
\hline $\mathbf{4 .}$ & Sun [28] & VeriFinger & EER: $6.79 \%$ \\
\hline $\mathbf{5 .}$ & Tao [30] & VeriFinger & EER: $5.83 \%$ \\
\hline $\mathbf{6 .}$ & Mishra [23] & ITIS & EER: $5 \%$ \\
\hline $\mathbf{7 .}$ & Proposed & & EER: $2 \%$ approx. \\
\hline
\end{tabular}

If we compare the union of minutiae coordinates and orientation angles of five thumbprints of Servesh with a randomly selected thumbprint of Servesh then the match percentage increases to $90 \%$ whereas after comparing the union of five thumbprints of Servesh with a new thumbrint of Dhermesh, we observed that the match percentage is in the range of $20 \%$ to $25 \%$. Similar results are obtained after comparing the union of thumbprints of Dhermesh with randomly selected thumbprints of Servesh and Dhermesh.

Finally, we found that either we take the union of six or seven or eight thumbprints of a person the matching percentage for a new thumbprint of the same person is approximately $90 \%$ or for a different person it is approximately $25 \%$. Thus, we are convinced at this point that the union of six thumbprints for a person of an identical twin pair can give the best matching result.

\subsection{Comparing the Result of Proposed Technique with Standard Methods}

We compared 50 identical twins of FVC datasets and we found that the proposed minutiae fusion based method is able to identify all the twins excluding one pair for which we did not get appropriate matching percentage. The proposed method is compared with existing identical twins thumbprint verification methods and the outcome is presented in table 6 .

\section{Conclusions}

In this research paper we have introduced a novel minutiae coordinates and orientation angles fusion based union set technique for thumbprint identification of identical twins. The proposed method is successfully identifying identical twins for self generated dataset and FVC datasets. The proposed method has proved that the dissimilarities between thumbprints images of identical twins are above $80 \%$ for self generated and standard (FVC2004, FVC2006) datasets.

The proposed method uses approximately $0.7 \mathrm{~KB}$ to $1.0 \mathrm{~KB}$ memory to store a thumbprint image of $55 \mathrm{~KB}$ to $60 \mathrm{~KB}$. The minutiae union set of a person requires $2.5 \mathrm{~KB}$ to $3.5 \mathrm{~KB}$ memory for storing minutiae features of six different samples which were scanned at a fixed time interval. 
The researchers can use left and right thumbprints fusion based soft computing technique for identifying identical twins. The socio behavioral features of identical twins can be combined with minutiae fusion based techniques for improving the matching results of identical twins identification methods.

\section{Acknowledgments}

The researchers are thankful to Professor (Dr.) Ram Pal Singh (Director, Birla Institute of Technology Ranchi, INDIA - Allahabad Campus) for his kind support in completing this research work. The researchers are thankful to the Vice Chancellor, Birla Institute of Technology Ranchi, India, for creating academic and research oriented environment at the institute.

The research team is thankful to Dr. R. Sukesh Kumar (Professor \& Head, Dept. of Computer Sc. \& Engg., B.I.T. Mesra, Ranchi) and Dr. G. Sahu (Professor \& Head, Dept. of I.T., B.I.T. Mesra) for encouraging us to write quality research papers.

\section{References}

[1] Bazan J.,Skowron A., "Perception Based Rough Set Tools in Behavioural Pattern Identification", Fundamenta Informaticae - New Frontiers in Scientific Discovery, Vol. 75, No. 1-4, pp. 27-47, 2007.

[2] Bennet D.,Peru mal S.A., "Fingerprint: DWT, SVD Based Enhancement and Significant Contrast for Ridges and Valleys Using Fuzzy Measures", Journal of Computer Science And Engineering, Vol. 6, No. 1, pp- 28-32, 2011.

[3] Chain T. P, Yau Wei-Yun, and Jiang X, "TokenBased Fingerprint Authentication", Recent Patents on Computer Science, Vol. 2, pp. 50-58, 2009.

[4] Chen X., Tian J., Yang X., and Zhang Y., "An Algorithm for Distorted Fingerprint Matching Based on Local Triangle Feature Set", IEEE Transactions on information forensics and security, Vol. 1 , No. 2, pp. 169-177, 2006.

[5] Chen X., Tian T., and Yang X., "A New Algorithm for Distorted Fingerprints Matching Based on Normalized Fuzzy Similarity Measure", IEEE Transactions on image processing, Vol. 15 No. 3, pp. 767-776, 2006.

[6] Han Y, Ryu C, Moon J, Kim H, and Choi H., "A study on evaluating the uniqueness of fingerprints using statistical analysis", Information Security and Cryptology-ICISC04, pp. 467 - 477, 2004.

[7] Homem N., and Carvalho J.P., "Mobile Phone User Identification with Fuzzy Fingerprints",
Advances in Intelligent System Research, Vol. 1, No. 1, pp. 860-867, 2011.

[8] http://bias.csr.unibo.it/fvc2004/ Last Accessed On: June 2012.

[9] http://bias.csr.unibo.it/fvc2006/ Last Accessed On: June 2012.

[10] Iancu, Constantinescu N., and Colhon M., "Fingerprints identification using a Fuzzy Logic System", Int. J. of computers Communications \& control, Vol. 5, No. 4, pp. 525-531, 2010.

[11] Indi S.T., and Raut D.S., "Biometric Feature Based Person Unique Identification System", International Joumal of Computer Application, Vol. 51, No. 13, pp. 7-12, 2012.

[12] Jain A.K., Ross A., and Prabhakar S. "An Introduction to Biometric Recognition", IEEE Transaction on Circuit and System for video technology, Vol. 14, No. 1, pp. 4-20, 2004.

[13] Jain A.K., Prabhakar S., and Pankanti S., "On the Similarity of identical twin fingerprint", Pattern Recognition, Vol. 35, pp. 2653-2663, 2002.

[14] Jain A. K., Feng J., and Nandakumar K., "Fingerprint Matching", Computer, Vol. 43, No. 2, pp. 36-44, 2010.

[15] Josphineleela R., and Ramakrishnan M., "An Efficient Automatic Attendance System Using Fingerprint Reconstruction Technique", International Journal of Computer Science and Information Security, Vol. 10, No. 3, pp. 1-6, 2012.

[16] Jung D.W., and Park R.H. "Robust Fingerprint Identification Based on Hybrid Pattern Recognition Methods", A Book of World Scientific pp. 1-26, 2001. Available Online On: www.worls cientific.co m/worlds cibook/10.1142/48 71

[17] Krishneswari K., and Arumugam S., "Multimodal Biometrics using Feature Fusion", Journal of Computer Science, Vol. 8, No. 3, pp. 431-435, 2012.

[18] Kondekar M.H, Kulkarni U.V., and Krishna Kanth B.B.M., "Extended Fuzzy Hyperline Segment Neural Network for Fingerprint Recognition", International Journal of Biometrics and Bioinformatics, Vol. 5, No. 3, pp. 162-171, 2011.

[19] Kulkarni J.V., Patil B.D., and Holambe R.S., "Orientation Feature for fingerprint matching ", Pattern Recognition, Vol. 39, pp. 1551-1554, 2006.

[20] Leung M., Engeler W., and Frank P. , "Fingerprint Image Processing Using Neural Network", Computer and comm. System, IEE TENCON 90, pp. 582-586, 1990. 
[21] Meva T.D., Kumbharana C.K., and Kothari A.D., "The Study of Adoption of Neural Network Approach in Fingerprint Recognition", International Joumal of Computer Application, Vol. 40, No. 11, pp. 8-11, 2012.

[22] Mishra K. N., Srivastava P. C., Agrawal Anupam, Tripathi V., and Gupta V.," A Framework Towards Using Eigen Iris, Minutia Thumb and DNA Sequence Features For Personal Identification", International Journal of Information Acquisition, Vol. 8, No. 3, pp. 197225, 2011.

[23] Mishra K. N., Srivastava P. C., Agrawal Anupam, Tripathi V., and Garg R., "Minutiae Distances and Orientation Fields Based Thumbprint Identification of Identical Twins", International Journal of Image Graphics and Signal Processing, Vol. 5, No. 3, pp. 51-59, 2013.

[24] Mishra K. N., Srivastava P. C., Agrawal Anupam, Ojha P., and Garg R., "Fingerprints, Iris and DNA Features Based Multimodal Systems: A Review", International Journal of Information Technology and Computer Science, Vol. 5, No. 2, pp. 88-111, 2013.

[25] Mudholkar S.S., Shende P.M., and Sarode M., "Biometrics Authentication Technique For Intrusion Detection System Using Fingerprint Recognition”, International Journal of Computer Science, Engineering and Information Technology, Vol. 2, No. 1, PP. 57-65, February 2012.

[26] Patil D.S., and Patil S.A., "Fingerprint recognition using minutia matching", World Journal of Science and Technology, Vol. 2, No. 4, pp. 178181, 2012.

[27] Srihari Sargur N., Srinivasan H., and Fang G., "Discriminability of Fingerprints of Twins", Journal of Forensic Identification, Vol. 58, No. 1, pp. 109-127, 2008.

[28] Sun Zhenan, Paulino A., Feng J., and Chai Z. "A Study of Multi-biometric Traits of Identical Twins", Proc of SPIE, Biometric Technology for Human Identification VII, pp. 7667 - 27, 2010.

[29] Tan X, and Bhanu B., "Fingerprint matching by genetic algorithms", Pattern Recognition, Vol. 39, pp. 465-477, 2006.

[30] Tao X., Chen X., Yang X., and Tian J., "Fingerprint Identification with Identical Twin Fingerprints", PLoS One, Vol. 7, No. 4, e35704, (doi:10.1371/journal.pone.0035704), 2012.

[31] Vatsa M., Singh R., Noore A., and Singh S.K., "Quality Induced Fingerprint Identification Using Extended Feature Set", In Proceedings of IEEE Conference on Biometrics: Theory, Applications and Systems, pp. 52-61, 2008.
[32] Velamuri R., and Thompson S.G., "Fingerprint recognition Using Fuzzy Inference Techniques", A report submitted by University of Texas, pp. 1-9, 2006.

\section{Authors' Profiles}

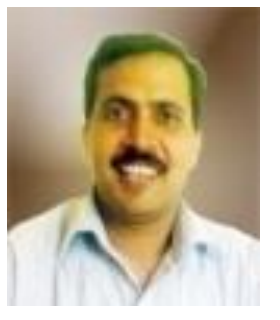

Kamta Nath Mishra was born on August 15, 1973 in Kushinagar district of Uttar Pradesh, INDIA. $\mathrm{He}$ received his Bachelor of Science (B.Sc., Maths) degree from University of Gorakhpur, Gorakhpur, INDIA, in 1991, and Master of Computer Application (MCA) degree from Madan Mohan Malviya Engineering College, Gorakhpur, U. P., INDIA in 1996. Mr. Mishra completed his M.S. (Software Systems) degree from Birla Institute of Technology and Science (BITS) Pilani, INDIA in 2003. Currently, he is working as an Assistant Professor at Dept. of CS\&E, B.I.T. Mesra, Ranchi, INDIA (Allahabad Campus) since August 2009, and pursuing Ph.D. from the same Institute in association with Indian Institute of Information Technology, Allahabad.

Mr. Mishra has worked as a faculty member in the department of Computer Science, University of Sebha, Sebha, LIBYA, from October 2006 to July 2009. He was a senior lecturer at B.I.T. Mesra, Ranchi, INDIA (Noida Campus) from July 2004 to September 2006. $\mathrm{Mr}$. Mishra has worked as a senior project engineer ( $\mathrm{Sr}$. Lecturer grade) from September 2003 to June 2004 and project engineer (Lecturer grade) from Septe mber 2000 to August 2003, in Centre for Development of Advanced Computing (Ministry of Communication \& IT, Govt. of India) Noida, Uttar Pradesh. Before joining CDAC, Mr. Mishra worked as a lecturer in CS\&E department at Krishna Institute of Engineering \& Technology (KIET), Ghaziabad, INDIA, from July 1998 to August 2000. His research interest includes Biometric Systems, Image Processing, and Analysis of Algorithms. Mr. Mishra is a professional member of IEEE Biometric Society USA, and ACM, USA.

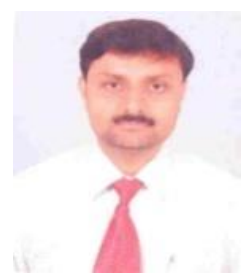

Prakash Chandra Srivastava received a Master degree in mathematics from University of Allahabad, INDIA in 2001, and Ph.D. degree from University of Allahabad, INDIA in 2009. He is an Assistant Professor at Birla Institute of Technology Ranchi, INDIA (Allahabad Campus). His research interest includes functional analysis and Software Measurement Methods. 


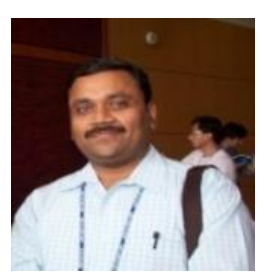

Anupam Agrawal is presently working as a Professor of Computer Science and Information Technology at Indian Institute of Information Technology Allahabad (IIIT-A). Before joining IIIT-A in the year 2000, he was working as scientist 'D' at DEAL, DRDO, Govt. of India, Dehradun. He received his M.Tech. degree in Computer Science and Engineering from Indian Institute of Technology Madras, Chennai and Ph.D. degree in Information Technology from Indian Institute of Information Technology Allahabad (in association with Indian Institute of Technology, Roorkee). He was a postdoctoral researcher at the Department of Computer Science \& Technology, University of Bedfordshire (UK) during which he had contributed significantly in two major European projects. His research interests include Computer Vision, Image Processing, Visual Computing, Soft- Computing and Human-Computer Interaction. He has more than 75 publications related to these areas in various international journals and conference proceedings, and has co-authored one book. He is on the review board for various international journals including IEEE, Springer, MDPI, Taylor \& Francis and Elsevier. He is currently serving as a Principal Investigator of an international (Indo-UK) Project. He is a member of ACM (USA), senior member of IEEE (USA) and a fellow of IETE (India). He is also serving as Chairman of the ACM Chapter at IIIT-A.

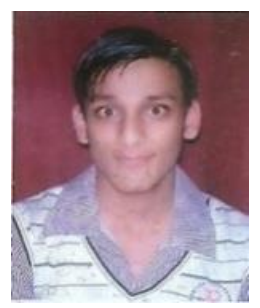

Rishu Garg received a Bachelor of Computer Application (BCA) degree from Rohilkhand University Bareilly, INDIA in 2010 and he is currently pursuing Master of Computer Applications (MCA) degree from Birla Institute of Technology, Ranchi, INDIA

(Allahabad Campus). His research interest includes fingerprints based identifications methods and Image Processing.

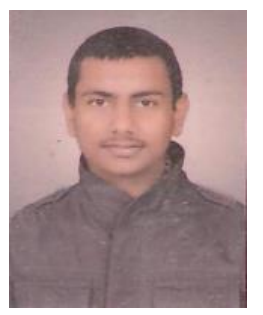

Ankur Singh received a Bachelor of Computer Application (BCA) degree from Birla Institute of Technology, Ranchi, INDIA (Allahabad Campus), in 2010 and he is currently pursuing Master of Computer Applications (MCA) degree from same Institute. His research interest includes Biometric Authentication Techniques based on thumb print recognition.
How to cite this paper: Kamta Nath Mishra, P. C. Srivastava, Anupam Agrawal, Rishu Garg, Ankur Singh,"Minutiae Fusion Based Framework for Thumbprint Identification of Identical Twins", International Journal of Intelligent Systems and Applications(IJISA), vol.6, no.1, pp.84-101, 2014. DOI: 10.5815/ijisa.2014.01.10 